\title{
Fingerprint Database Enhancement by Applying Interpolation and Regression Techniques for IoT-based Indoor Localization
}

\author{
Dwi Joko Suroso ${ }^{1}$, Farid Yuli Martin Adiyatma ${ }^{2}$, Panarat Cherntanomwong ${ }^{1 *}$, \\ Pitikhate Sooraksa ${ }^{1}$ \\ ${ }^{1}$ School of Engineering, King Mongkut's Institute of Technology Ladkrabang, 1, Soi Chalongkrung 1, Bangkok 10520, Thailand \\ ${ }^{2}$ Department of Nuclear Engineering and Engineering Physics, Universitas Gadjah Mada, Jl. Grafika No. 2, Yogyakarta 55281, Indonesia
}

\begin{abstract}
Most applied indoor localization is based on distance and fingerprint techniques. The distance-based technique converts specific parameters to a distance, while the fingerprint technique stores parameters as the fingerprint database. The widely used Internet of Things (IoT) technologies, e.g., Wi-Fi and ZigBee, provide the localization parameters, i.e., received signal strength indicator (RSSI). The fingerprint techniqueadvantages overthe distance-based method as it straightforwardly uses the parameter and has better accuracy. However, the burden in database reconstruction in terms of complexity and cost is the disadvantage of this technique. Some solutions, i.e., interpolation, image-based method, machine learning (ML)-based, have been proposed to enhance the fingerprint methods. The limitations are complex and evaluated only in a single environment or simulation. This paper proposes applying classical interpolation and regression to create the synthetic fingerprint database using only a relatively sparse RSSI dataset. We use bilinear and polynomial interpolation and polynomial regression techniques to create the synthetic database and apply our methods to the 2D and 3D environments. We obtain an accuracy improvement of $0.2 \mathrm{~m}$ for $2 \mathrm{D}$ and $0.13 \mathrm{~m}$ for $3 \mathrm{D}$ by applying the synthetic database. Adding the synthetic database can tackle the sparsity issues, and the offline fingerprint database construction will be less burden.
\end{abstract}

\section{Keywords:}

Indoor Localization;

Internet of Things; Zigbee;

Fingerprint Technique;

Fingerprint Database;

Interpolation; Regression; Polynomial.

\section{Article History:}

Received: 09 September 2021

Revised: $\quad 31$ December 2021

Accepted: 05 January 2022

Published: 16 January 2022

\section{1- Introduction}

Internet of Things (IoT) technology advancement has been flourishing in the last decades [1, 2]. Its implementation cannot be excluded from our everyday life. One of the features that are frequently used is positioning. As the wellestablished global positioning system (GPS) is most used for positioning, especially in the outdoor environment, it fails to give the proper accuracy positioning in the indoor environment [3, 4]. Therefore, some IoT-based technologies, i.e., Wi-Fi [5, 6], ZigBee [7], Bluetooth Low Energy (BLE) [8], Ultra-wideband (UWB) [4, 9], Radio Frequency Identification (RFID) [10], can be applied for Indoor Positioning Systems (IPS) instead of GPS's utilization indoor [5]. More commonly stated as indoor localization, IPS can be achieved by applying the technologies mentioned above and specific methods or techniques.

Wireless sensor networks (WSNs), also based on IoT technology, are known as one of the most used for indoor localization implementation $[4,11,12]$. This paper considers the low-cost and straightforward implementation of WSNsbased indoor localization using the ZigBee standard [13]. Compared to other technologies such as Wi-Fi, the ZigBee can have a more flexible setup and deployment of the WSNs system. On the other hand, indoor localization techniques include algorithms to identify the target's location based on several signal properties based on the technologies offered.

\footnotetext{
* CONTACT: panarat.ch@kmitl.ac.th

DOI: http://dx.doi.org/10.28991/esj-2021-SP1-012
}

(C) 2020 by the authors. Licensee ESJ, Italy. This is an open access article under the terms and conditions of the Creative Commons Attribution (CC-BY) license (https://creativecommons.org/licenses/by/4.0/). 
ZigBee standard provides some signal properties that can be used for localization algorithm, i.e., Received Signal Strength Indicator (RSSI) and Link Quality Indicator (LQI) [14, 15]. The advantages of RSSI include simple to obtain and straightforward implementation without additional hardware installation. However, the drawback of RSSI properties is that the signal somehow fluctuates and varies due to time. For LQI, the additional hardware to record the data transmission may require, and its implementation is not typical [16].

The indoor localization techniques are generally divided into two, as the range or distance becomes the center of concern. The distance-based and the distance-free techniques are widely applied for indoor localization techniques [17]. Distance-based techniques solely rely on distance measurement to estimate the location. This distance can be from the conversion parameter of signal power, i.e., RSSI mentioned before, or angle or time information from the signal transmission. One advantage of this range-based technique is that if the algorithm has received an excellent distancesignal properties conversion, the localization can be done straight away without pre-processing or databasing. These advantages also depend on the kind of signal properties used for distance conversion. However, the range-based technique will yield a high error if unreliable signal properties are received and poorly converted as the distance parameter [18].

The distance-free indoor localization technique, on the other hand, has advantages in reducing the effect of this signal fluctuation caused by the multipath effect in the indoor environment by collecting the spatial information of this signal to record as the database. The common distance-free technique in indoor localization is fingerprinting [19]. The fingerprinting technique requires two phases for the localization, the first phase is called the offline phase, and the second phase is the online phase [20]. In the offline phase, the necessary information related to the spatial information of the area of interest is recorded as the offline fingerprint database. The fingerprint database consists of the signal properties information related to a specific location on the designated grids inside the area of interest: the denser these grids, the more accurate and precise later for the online phase or the localization process. After the data or localization parameters are measured and stored in the database acquisition, the online phase, in which the target or object sends the signal parameter to the system and receive the same parameters as recorded in the database, the pattern matching algorithm will work by comparing the target's signal parameters to those in database. This algorithm then concludes that the target or object belongs to a particular position with similar spatial information recorded in the fingerprint database.

However, the fingerprint technique has drawbacks in the offline database construction phase [21, 22]. It takes much effort, high cost, and sometimes has complexity or scalability issues when the applied indoor environment is enormous. There are several attempts to reduce the drawbacks, i.e., constructing the artificial grids by applying classical to machine learning-based techniques and reducing the database complexity by employing some compression algorithm [23]. The effort in signal point-of-view enhances the signal parameters using the dedicated filter to fight the signal fluctuations and implements several clustering techniques to remove the data outliers and improve localization accuracy [24, 25]. For all of this method, as the author is concerned, there are still open challenges in implementing accurate and straightforward fingerprint database enhancement, i.e., simple interpolation and regression techniques [26-28].

Some proposals that applied the interpolation techniques to tackle the database sparsity have been published in [29, 30]. The most used interpolation technique for fingerprint-based indoor localization is the Kriging technique. The authors in [31] showed that the Kriging for RSSI, especially in inaccessible areas, can be covered and enhance the overall fingerprint database. Another approach of the database enhancement by path-loss model-based interpolation is available in [28, 32], the crowdsourcing method [33], Spatio-temporal similarity [34], and clustering-based and interpolation on [35]. However, most of these approaches have limitations on algorithm complexities. Furthermore, some issues related to the advancement parameter, e.g., Channel State Information (CSI) and its complexity, appear and become the drawbacks of applying this parameter. Thus, as far as our concerns, the simple yet straightforward implementation of interpolation and regression technique is not yet considered - especially when using WSNs-based as the system's core. By utilizing RSSI following the log-loss distance, it views the linear assumption relationships between signal strength and the distance. By considering this, the interpolation and regression can be established as the power-distance relationship in the applied environment. In addition, we consider conducting an actual measurement campaign in our approach. The approach is the algorithm development in simulation or theory and actual implementation both for twodimensional (2D) and three-dimensional (3D) by using the ZigBee standard as the core of the WSNs system. Our original achievements are enhancing the density database by using a relatively sparse actual measurement database by applying basic interpolation and regression. Our approach is relatively simple compared to previously mentioned publications. Our significant difficulties to overcome is the RSSI fluctuation in some parts of fingerprint position because of the nature of the environment, i.e., near the edge, enormous metal material, and unbalanced obstruction in the environment, making the synthetic database challenging to assure.

The fingerprint technique for both environments is applied, and the offline database fingerprint is obtained by the area of interest $5 \times 5 \mathrm{~m}^{2}$ for the 2D environment. We utilize the bookshelf as the $3 \mathrm{D}$ environment, assuming several floor applications in a multi-story building in the same environment as the 2D settlement. We design the database grid of $1 \times$ $1 \mathrm{~m}$ for the $2 \mathrm{D}$ and $22 \times 12.5 \times 35 \mathrm{~cm}$ for the $3 \mathrm{D}$ environment. The $\mathrm{cm}$-scale is to observe how our interpolation and 
regression algorithm can work well and acceptable in such a short distance. The pattern matching used is the classical minimum Euclidean distance (MED) to know-how is the difference between the predicted and the actual RSSI values. The tiniest error yield from the target-database comparison is assigned as the target's location. From the context we have explained and by proposing the method for actual implementation, we would like to highlight the contribution of our proposed method as follows:

- Implementing the interpolation and regression technique for database enhancement in fingerprint-based indoor localization considering 2D and 3D environments.

- We compare the interpolation and regression techniques to observe the best use of the technique for the case of 2D and $3 \mathrm{D}$ environments.

We present the structure of this paper as follows; in the first part, we discuss the introduction and background stated the context and importance of our proposal. In the second part, we present the indoor localization technologies and techniques and the fingerprint technique's comprehensive explanation. For the third part, we detail the material and method, including the ZigBee-based WSNs system, interpolation, regression technique we propose to implement, pattern matching, performance metrics, and the measurement system and setup. Results and discussion will then be presented in the fourth part. Finally, we will conclude our findings and discuss our proposal limitations and plan of our near-future works.

\section{2- Indoor Localization}

This part will discuss indoor localization, including its technology, distance measurement, localization, and fingerprint technique.

\section{2-1-Indoor Localization Technologies}

Indoor localization can act as an "indoor GPS," and some technologies have been introduced to develop indoor localization research. The technologies include radio frequency (RF)-based and other, i.e., mechanical, optical/light wave, acoustic wave, and vision-based, also attract the research further. However, RF-based researches are most common and widely implemented. Table 1 shows the most-used RF-based indoor localization technologies and their features [17].

Table 1. RF-based indoor localization technology.

\begin{tabular}{cccc}
\hline Technology & Accuracy $(\boldsymbol{m})$ & Range $(\boldsymbol{m})$ & Power $(\boldsymbol{W})$ \\
\hline GPS & $1-20$ & global & 500 \\
RFID & 1 & $1-50$ & $0.02-0.3$ \\
Wi-Fi & $1-5$ & $<100$ & $0.5-1$ \\
UWB & $<0.3$ & $<300$ & 0.03 \\
BLE & 1 & $<10$ & 0.001 \\
ZigBee & $1-5$ & $<30$ & $0.02-0.04$ \\
\hline
\end{tabular}

In this paper, we consider applying the ZigBee technology because of several reasons:

- By comparing the accuracy of other technologies in Table 1, the ZigBee system can achieve an accuracy of $1-5$ m. UWB technology can achieve better accuracy than ZigBee. However, implementation of UWB need additional hardware, and to achieve $<0.3 \mathrm{~m}$ accuracy, several advances and complicated algorithm are needed, e.g., Time-ofArrival (ToA), Ranging Time (RT).

- Observing the range properties of ZigBee technology, the $<30 \mathrm{~m}$ can cover almost all indoor environments, the scalability issues can be solved.

- Power consumption is also low compared to other technologies; only RFID and BLE give the same or less than ZigBee. However, the range of BLE is relatively short, while the RFID technology also requires the line-of-sight (LoS) communication between tags and the RFID reader.

ZigBee standard is the IEEE 802.15.4 standard working in industrial, scientific, and medical (ISM) bands similar to Wi-Fi in $2.4 \mathrm{GHz}$. In the measurement system and setup, we will explain how to reduce the effect of the signal interference with Wi-Fi.

\section{2-2-Distance Measurement Technique}

Figure 1 shows the several distance measurement or signal properties used in indoor localization. 


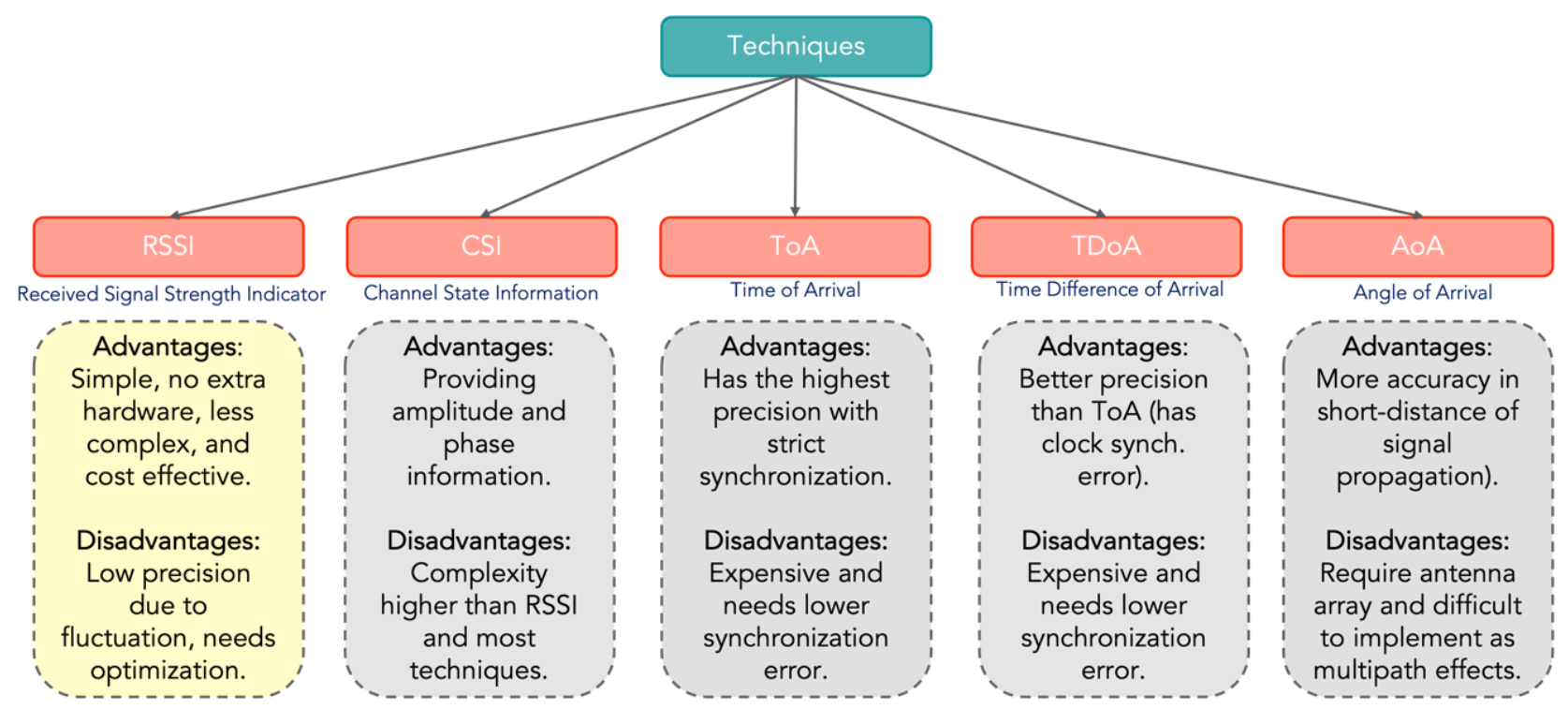

Figure 1. Distance measurement technique.

There are some advantages and disadvantages of each distance measurement technique. However, we can note from the illustration that the RSSI parameter has the most prospective implementation, especially in our approach. First, it has advantages in the straightforward implementation, the second there are disadvantages point that becomes a focal point, especially for the database enhancement in our proposal. RSSI is defined as the received power, and it follows the log-loss distance model [36]:

$$
\operatorname{RSSI}(\mathrm{dBm})=A-10 \cdot n \cdot \log _{10}\left[\frac{d_{0}}{d}\right]
$$

RSSI in $\mathrm{dBm}$ related to the distance, $d$, is equal to the $A$, power in $\mathrm{dBm}$ of reference distance, $d_{0}$ subtracted by the path loss exponent, $n$, multiply the $\log$ distance of $d_{0}$ divided by the $d$; generally $d_{0}$ is $1 \mathrm{~m}$. The values of $n$ can be measured by empirical in the particular indoor environment used. In our approach, we collected the RSSI values from the ZigBee standard without considering the RSSI-distance conversion.

\section{2-3-Indoor Localization Methods}

Figure 2 shows several basic methods applied in indoor localization systems.
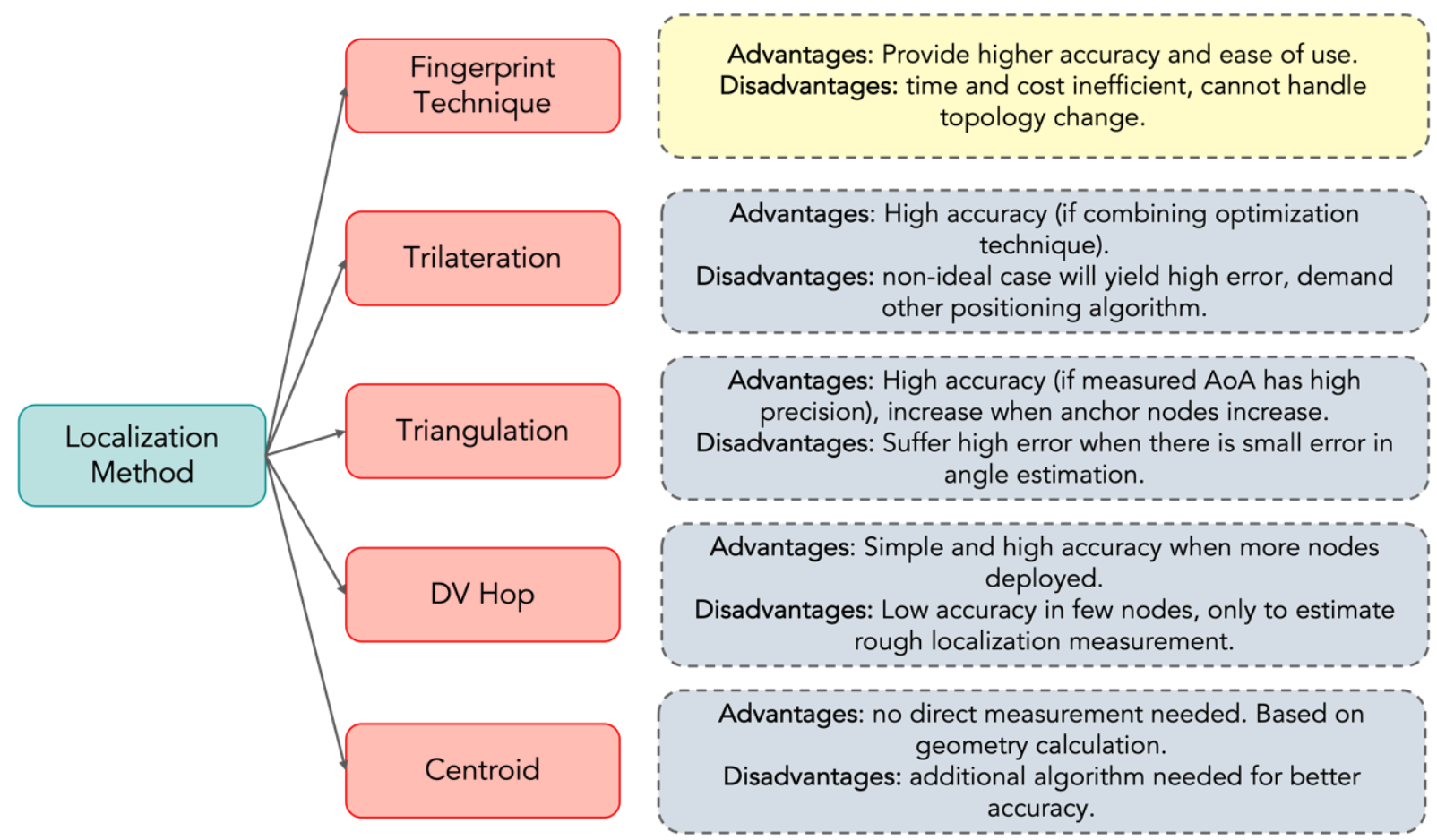

Figure 2. Localization methods. 
The fingerprint technique gives advantages when there is no need for distance-parameter conversion. Furthermore, the fingerprint technique gives better accuracy than several range-based techniques, i.e., triangulation or trilateration, min-max, and iRingLa compared based on RSSI as localization parameters [37]. The other techniques can also yield high accuracy but need additional support in the algorithm, precise clocking, accurate angle estimation, and the high number of nodes or beacons used for positioning. For previously mentioned advantages of fingerprint technique, however, there is a drawback of fingerprint technique, especially in the offline database construction process, with the burden of the cost and time inefficient, moreover, if there is an application in the large-scale indoor environment, the system will be more complex and needs more human resources. The trade-off of this technique is that if we do not have enough density of fingerprint database, the localization error will be high. On the contrary, when we need to have a very dense fingerprint database, multiple drawbacks will appear related to offline databasing. In this paper, we propose the database enhancement in tackling the drawbacks of the fingerprint technique, especially in the database sparsity, by applying the interpolation and regression technique.

\section{2-4-Fingerprint Technique}

The fingerprint technique is similar to fingerprint pattern recognition in image processing. However, the terms fingerprint here refers to radio fingerprints' spatial information. In the previous section, we discussed the general definition of the fingerprint technique. We have also shown the disadvantages in the fingerprint technique implementation, especially in the database construction process. This section will discuss other related fingerprint techniques, their disadvantages, and solutions. The illustration of the fingerprint technique process can be depicted in Figure 3.

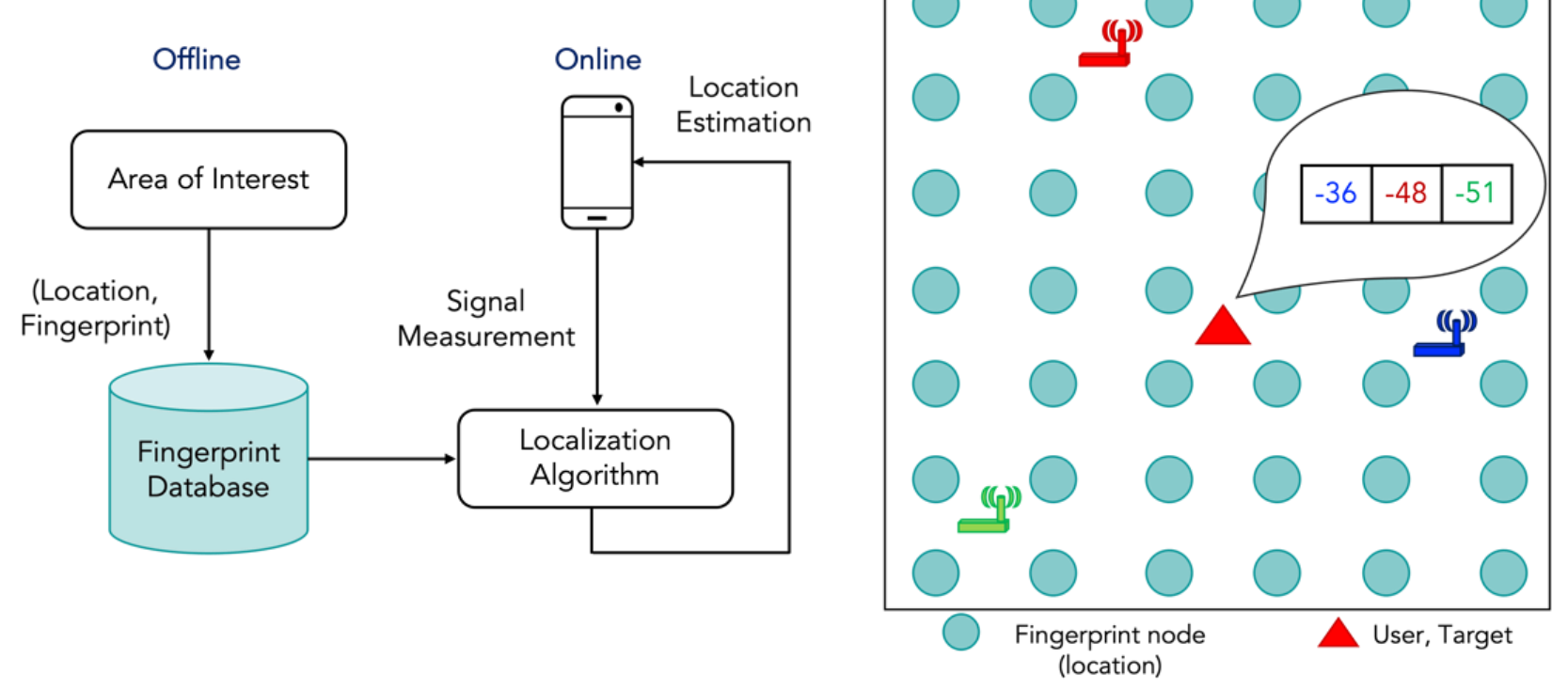

Figure 3. Fingerprint technique.

The fingerprint technique works by constructing the database with spatial information needed to estimate target or object position. This spatial information is the location of the fingerprint point with its corresponding parameter. In this Figure 3, the corresponding parameter is RSSI values from three reference nodes/beacons. The fingerprint technique applies the two phases; the first phase is the offline phase for storing the spatial information as the database, and the second is the signal measurement by an object to be localized by a localization algorithm or pattern matching algorithm. For our proposal, the detail of our fingerprint technique workflow is depicted in Figure 4.

The known fingerprint location depicted in Figure 3 shown in the RSSI-based fingerprint technique in Figure 4 as the FP Location 1, FP Location 2, .., FP Location $N$ will collect the RSSI from the reference nodes $i$ to $M$, with $i=$ $1,2, \ldots, M$ as the $F D_{i}, \ldots, F D_{M}$. After all, the fingerprint points and their corresponding RSSI values are stored in the database. The online phase is when the target calculates the RSSI values from the same $i$ to $M$ nodes and comparing to those in database by applying a pattern matching algorithm. In our proposal, we propose applying the minimum Euclidean distance (MED) algorithm to find the similarity between target parameter, $T$ and the $F D_{i}, \ldots, F D_{M}$ in the database. Once the $i^{\text {th }}$ fingerprint database, which has similar RSSI values, is successfully concluded, the correspondence location of this $i^{t h}$ database, the $i^{\text {th }}$ FP Location is estimated as the target or object location. 


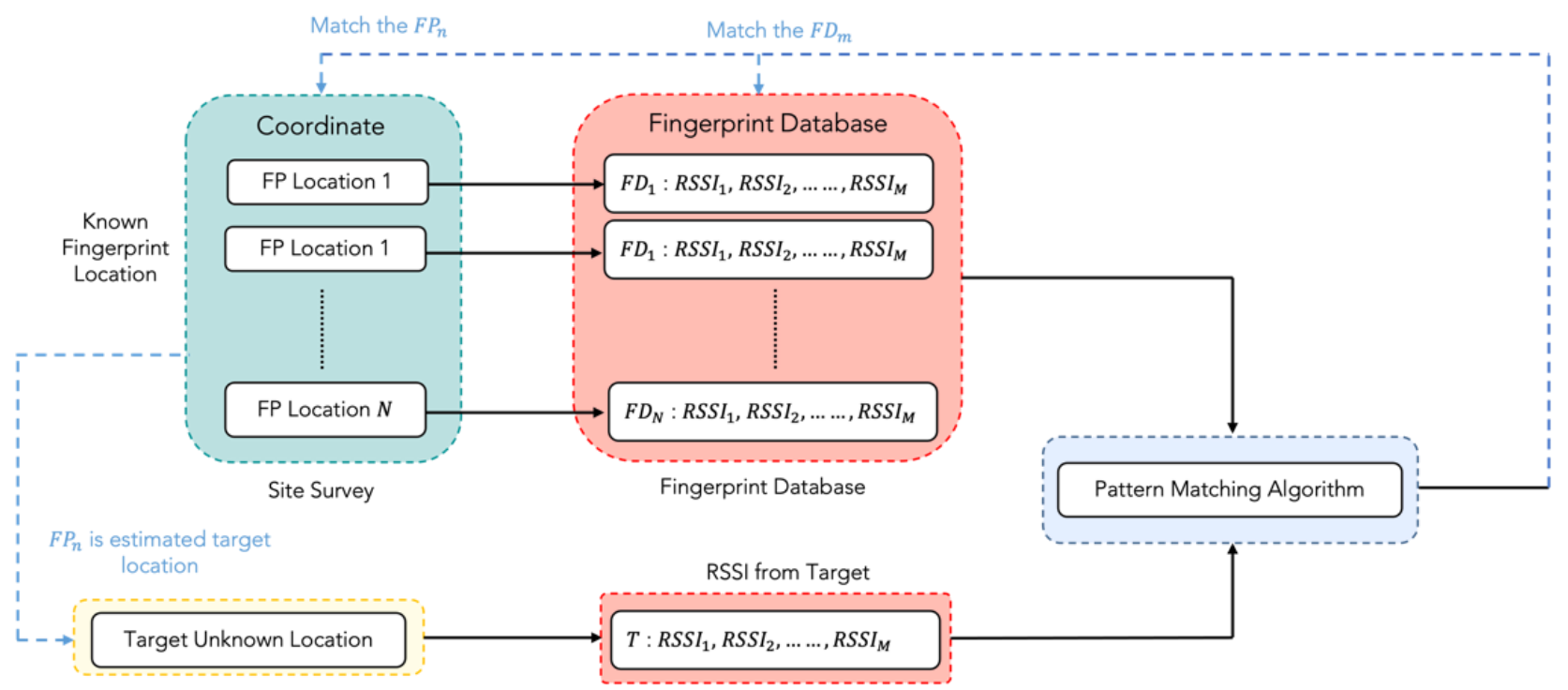

Figure 4. RSSI-based fingerprint technique.

The fingerprint technique performance metrics, i.e., accuracy and precision, are heavily dependent on the quality of the database. One of the factors is the density of the database. However, there will be more burden processes in the offline phase to get a denser database. Some challenges and their prospective solutions can be seen in Figure 5.

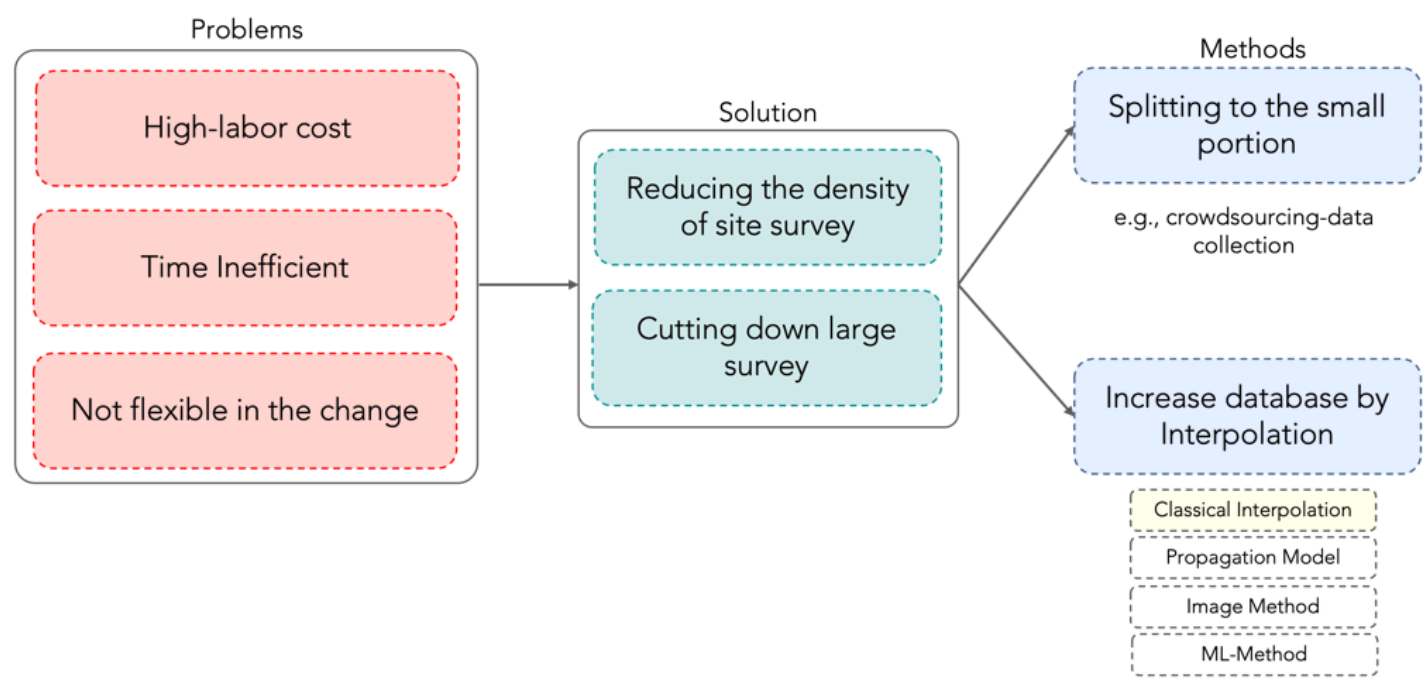

Figure 5. Challenges and prospective solutions of the fingerprint technique.

Cutting down extensive surveys or the database's density improvement can be one of the solutions [39]. Some proposals on the propagation model-based database enhancement have a drawback in the high cost of the channel measurement device and measurement campaigns [40-42]. The other approaches are by using the image method in which the grayscale image of the image processing method is applied to convert the RSSI values in the area of interest [38]. The imaging method is promising but highly complex in the super-resolution image conversion from the RSSI to grayscale image conversion. The ML-based method for database augmentation has abundantly proposed, and because of the threshold in the number of data required in some ML techniques, the complexity is also high [22, 43, 44]. As far as the author is concerned, there are few, or there is no attempt yet in using the classical interpolation technique for this database synthesis. Therefore, this paper proposes utilizing the classical interpolation and regression technique with low complexity and achieving acceptable performance results.

\section{3- Material and Methods}

\section{3-1- Wireless Sensor Networks (WSNs) using ZigBee Standard}

The ZigBee device, XBee-24ZB, is utilized for both the target and reference nodes in our WSNs setup. We apply the topology star for the localization system. Here, the sink node is the target located inside the area of interest, while the reference nodes are in the corners of the area of interest. Figure 6 shows the illustration of our WSNs based on ZigBee $[17,45]$. 


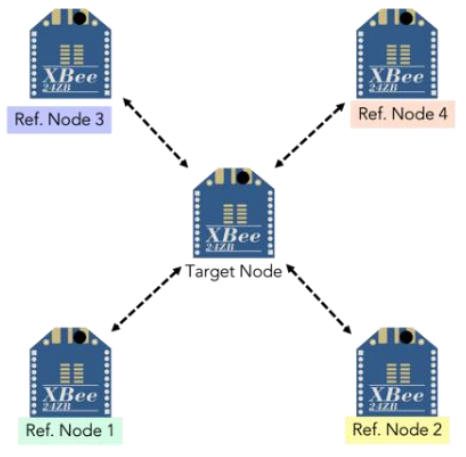

(a)

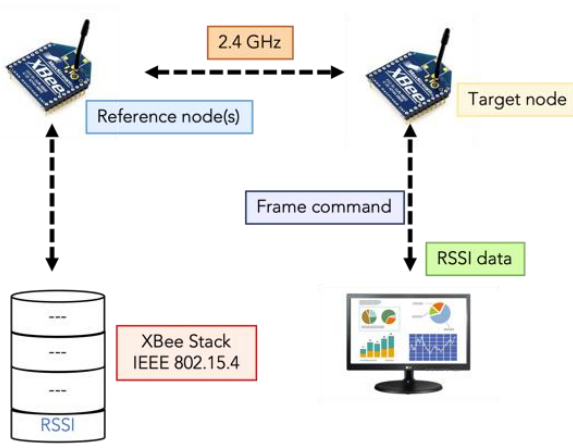

(b)

Figure 6. a) Topology star illustration, b) The RSSI acquiring process.

The RSSI acquiring process starts with request from the sink node/target to the reference nodes. The reference nodes then get the RSSI packet request by sending back the RSSI from the XBee stack to the sink node. The sink node then translates the packet and store the RSSI values to the storing device/personal computer.

\section{3-2-Interpolation and Regression Technique}

The interpolation technique predicts a point or a value between two or more known points/values. The basic interpolation techniques are divided into linear and polynomial interpolation. The linear interpolation technique predicts the values between two points, while interpolation can predict specific points using several known data points.

\section{Bilinear Interpolation Teukolsky et al. [46]}

- Take example of a simple linear interpolation for two data points, $y_{1}$ and $y_{2}$, as in Figure 7 .

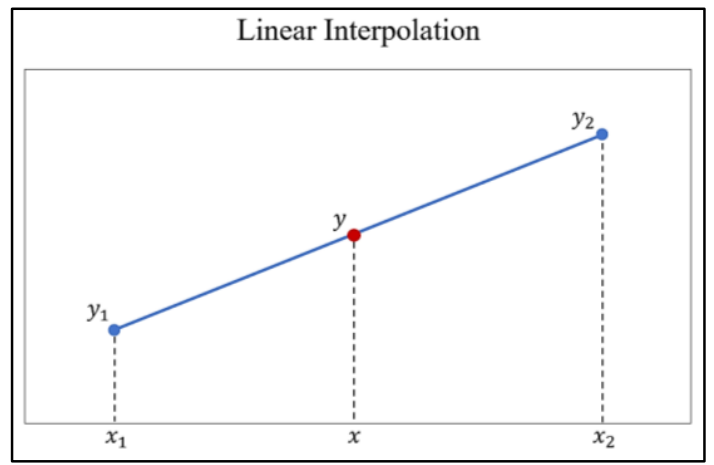

Figure 7. Linear interpolation illustration.

The $y$ between $y_{1}$ and $y_{2}$ can be predicted as in Equation 2;

$y=\frac{x-x_{1}}{x_{2}-x_{1}}\left(y_{2}-y_{1}\right)+y_{1}$

- Bilinear interpolation extends a linear interpolation on two cartesian coordinate axes, the $x$-axis and the $y$-axis. Suppose there are 4 points $\left(x_{1}, y_{1}\right),\left(x_{2}, y_{1}\right),\left(x_{2}, y_{2}\right)$, dan $\left(x_{1}, y_{2}\right)$, having functions of $f\left(x_{1}, y_{1}\right), f\left(x_{2}, y_{1}\right)$, $f\left(x_{2}, y_{2}\right)$, and $f\left(x_{1}, y_{2}\right)$. We can use these values to predict the $f(x, y)$ value at $(x, y)$ as illustrated in Figure 8 .

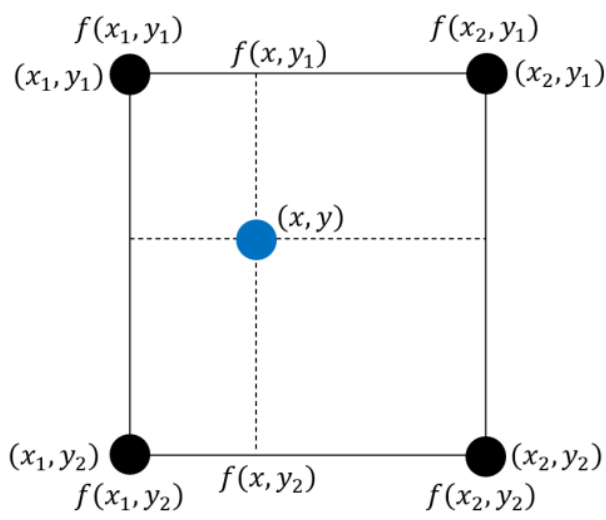

Figure 8. Bilinear interpolation illustration. 
The value at $(x, y)$ can be predicted by first, we first interpolate the $\mathrm{x}$-axis as

$f\left(x, y_{1}\right)=\frac{x-x_{1}}{x_{2}-x_{1}}\left(f\left(x_{2}, y_{1}\right)-f\left(x_{1}, y_{1}\right)\right)+f\left(x_{1}, y_{1}\right)$.

$f\left(x, y_{2}\right)=\frac{x-x_{1}}{x_{2}-x_{1}}\left(f\left(x_{2}, y_{2}\right)-f\left(x_{1}, y_{2}\right)\right)+f\left(x_{1}, y_{2}\right)$.

Thus, by using Equations 3 and 4, we can the proceed to y-axis interpolation by Equation 5 .

$(x, y)=\frac{y-y_{1}}{y_{2}-y_{1}}\left(f\left(x, y_{2}\right)-f\left(x, y_{1}\right)\right)+f\left(x, y_{1}\right)$.

\section{Polynomial Interpolation Kiusalaas (2013) [47]}

- If we have more than two data points, polynomial interpolation can predict the data points within the data range. Polynomial interpolation can also handle non-linear pattern data. Neville interpolation is one of the polynomial interpolation methods.

- Predicted value within the data range can be obtained using general equation shown in Equation 6.

$P_{k}\left[x_{i}, x_{i+1}, \ldots, x_{i+k}\right]=\frac{\left(x-x_{i+k}\right) P_{k-1}\left[x_{i}, x_{i+1}, \ldots, x_{i+k-1}\right]+\left(x_{i}-x\right) P_{k-1}\left[x_{i+1}, x_{i+2}, \ldots, x_{i+k}\right]}{x_{i}-x_{i+k}}$.

We can solve the Equation 6 by using Neville Method Settlement detailed in Table 2 and $k$ is the degree of the polynomial equation.

Table 2. Neville Method Settlement.

\begin{tabular}{ccccc}
\hline & $k=0$ & $k=1$ & $k=2$ & $k=3$ \\
\hline$x_{0}$ & $P_{0}\left[x_{0}\right]=y_{0}$ & $P_{1}\left[x_{0}, x_{1}\right]$ & $P_{2}\left[x_{0}, x_{1}, x_{2}\right]$ & $P_{3}\left[x_{0}, x_{1}, x_{2}, x_{3}\right]$ \\
$x_{1}$ & $P_{0}\left[x_{1}\right]=y_{1}$ & $P_{1}\left[x_{1}, x_{2}\right]$ & $P_{2}\left[x_{1}, x_{2}, x_{3}\right]$ & \\
$x_{2}$ & $P_{0}\left[x_{2}\right]=y_{2}$ & $P_{1}\left[x_{2}, x_{3}\right]$ & & \\
$x_{3}$ & $P_{0}\left[x_{3}\right]=y_{3}$ & & & \\
\hline
\end{tabular}

- There are three solution steps for solving the Neville interpolation.

1.First step: Performing the interpolation of degree 1 by using the Equation 7.

$P_{1}\left[x_{0}, x_{1}\right]=\frac{\left(x-x_{1}\right) P_{0}\left[x_{0}\right]+\left(x_{0}-x\right) P_{0}\left[x_{1}\right]}{x_{0}-x_{1}}$.

2. Second step: Performing the interpolation of degree 2 by using the Equation 8 .

$P_{2}\left[x_{0}, x_{1}, x_{2}\right]=\frac{\left(x-x_{2}\right) P_{1}\left[x_{0}, x_{1}\right]+\left(x_{0}-x\right) P_{1}\left[x_{1}, x_{2}\right]}{x_{0}-x_{2}}$.

3.Third step: Performing the interpolation of degree 3 by using the Equation 9.

$P_{3}\left[x_{0}, x_{1}, x_{2}, x_{3}\right]=\frac{\left(x-x_{3}\right) P_{2}\left[x_{0}, x_{1}, x_{2}\right]+\left(x_{0}-x\right) P_{2}\left[x_{1}, x_{2}, x_{3}\right]}{x_{0}-x_{3}}$.

\section{Polynomial Regression Ostertagová (2012) [48]}

- Polynomial regression is a multiple regression with one independent variable. In one variable polynomial regression equation shown as Equation 10, $x$ is expressed as an independent variable.

$$
y=a_{0}+a_{1} x_{i}+a_{2} x_{i}^{2}+\cdots+a_{n} x_{i}^{k}+e_{i}, \quad i=1,2, \ldots, k \text {. }
$$

\section{3-3-Pattern Matching and Performance Metrics}

We applied a simple Minimum Euclidean Distance (MED) to match the RSSI of the target with those in the database. To validate our localization system's accuracy, we used average distance error (ADE) from all errors of target positions in each scenario, both for 2D and 3D environments [5]. Nevertheless, first, we evaluate the RSSI error value between the actual and predicted RSSI form interpolation and regression techniques by Equation 11. This error will be presented in the results and discussion for only $2 \mathrm{D}$, as the RSSI maps from $2 \mathrm{D}$ will be easier to interpret. The absolute symbol 
shows the RSSI discrepancy on the values (because RSSI in dBm is in a negative form). The RSSI error is considered in some points of the $2 \mathrm{D}$ fingerprint database to ensure that the proposed method is visible to apply by observing these errors.

$$
R S S I \text { error }(\mathrm{dBm})=\mid R S S I \text { value }(\mathrm{dBm})_{\text {actual }}-R S S I \text { value }(\mathrm{dBm})_{\text {predicted }} \mid .
$$

MED as pattern matching utilizes the Euclidean distance to measure the distance from two points; in our case, the Euclidean distance is the distance of RSSI values in the database and RSSI values of the target. The tiniest error or the minimum error of RSSI by their Euclidean distance for specific fingerprint location is assigned as the target's location.

$$
\text { Euclidean distance }(m)=\sqrt{\left(R S S I_{\text {database }}-R S S I_{\text {target }}\right)^{2}} \text {. }
$$

After we obtain the predicted location based on the similarity of the target and database by the MED, we evaluate the accuracy by the error in the meter of the predicted and the actual target location. Thus, the system's performance metric in our proposal is how the error of the target location prediction, $x_{\text {predicted }}$ and $y_{\text {predicted }}$, is compared to the actual target location, $x_{\text {actual }}$ and $y_{\text {actual }}$. Suppose there are $L$ number of target locations, we utilized ADE as the performance metric, the mean value of all Euclidean distances for all target locations. If the position of the target node as $i=$ $1,2, \ldots, L$, we can express the ADE as in Equation 13 [49].

$A D E=\frac{1}{L} \sum_{i=1}^{L} \sqrt{\left(x_{\text {actual }, i}-x_{\text {predicted }, i}\right)^{2}+\left(y_{\text {actual }, i}-y_{\text {predicted }, i}\right)^{2}}$

\section{3-4-Methodology Flow Diagram}

We design the measurement by applying wireless sensor networks (WSNs)-based data collection. For the flow diagram, we divide into two flows; first flow is an interpolation confirmation step where some of the database points are replaced by the interpolated RSSI values, and the second flow is the interpolation implementation step where the synthetic database from interpolation and regression are combined and evaluated by the localization performance. We start with the confirmation step, where the preparation is to design the measurement for two-dimensional (2D) and threedimensional (3D) environments, including how we propose the grids of fingerprint and target locations in the area of interest.
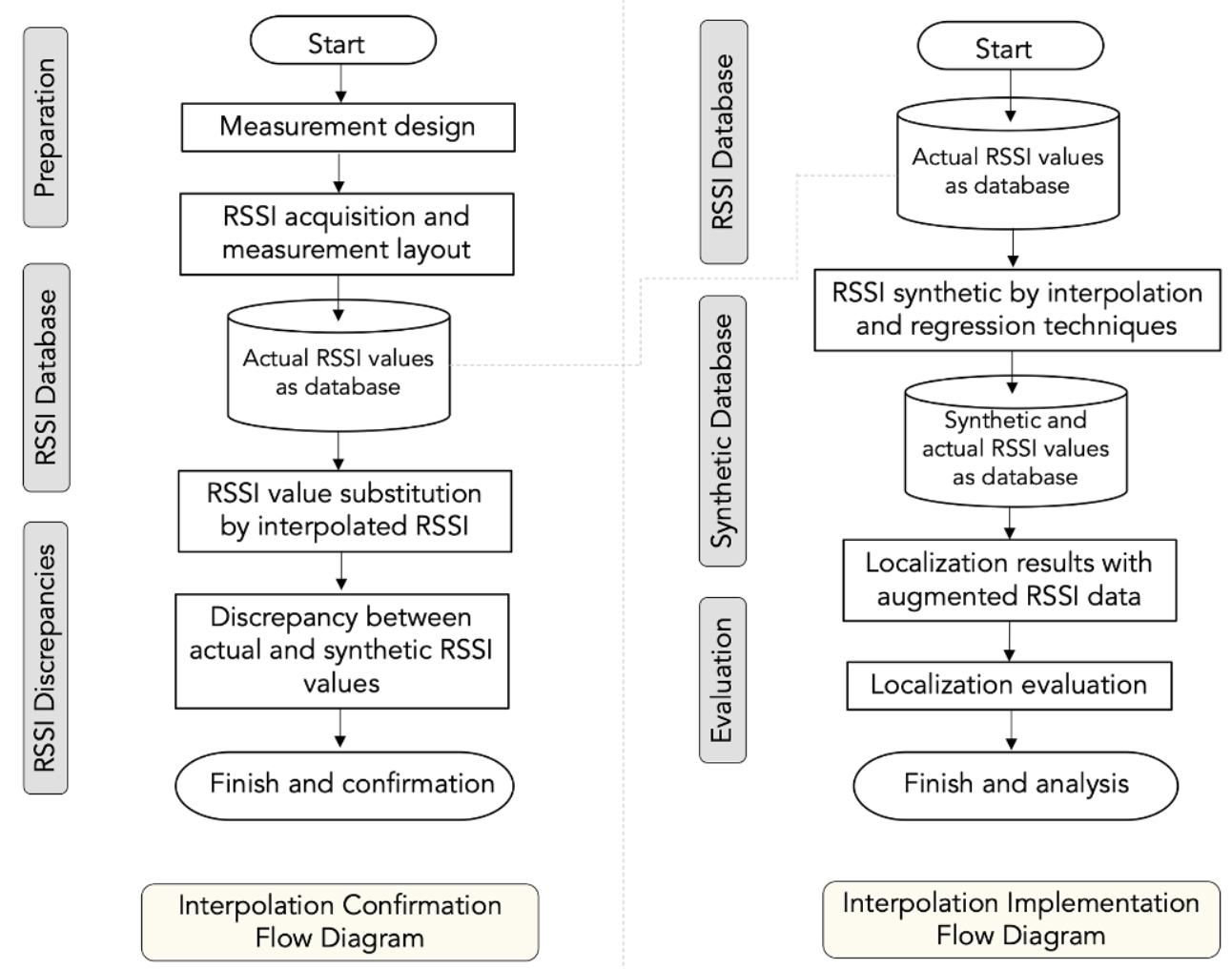

Figure 9. Flowchart of the research Methodology.

The RSSI data acquisition step uses the personal computer connected to the sink node (acts as the target node) and follows the star topology. We obtained the actual RSSI and stored it as the fingerprint database. The next step is to do 
interpolation in the same grid points as the substituted database to ensure the feasibility of using the interpolated data. After confirmation, the second flow uses the actual RSSI values for the database. We applied classical interpolation and regression methods to augment the denser data. We combine the actual and the synthetic database, then evaluate the localization results using the substituted RSSI in some points between and outside (in the case of the 3D environment) of the fingerprint database. The primary step is to make the database denser by applying interpolation and regression as our proposal. We evaluate and compare the localization results for interpolation and regression techniques for the synthetic database as we have the denser database. We discuss the results and conclude what we find.

\section{3-5-Measurement Campaign}

We consider the two 2D and 3D environments for indoor localization system deployment. The first environment is the lobby environment in our department, and we consider only a 2D scenario. For the 3D environment, we simulate the actual multi-story building by using a 4-level bookshelf for elevation parameters. The ZigBee devices are configured as reference nodes and target nodes; both acted as transceivers and were configured as a star topology.

\section{The 2D Measurement Campaign}

We conducted a measurement campaign for the $2 \mathrm{D}$ environment in the lobby of our building for a $5 \times 5 \mathrm{~m}^{2}$ area of interest. The layout and the actual setup of the measurement campaign for the 2D case are shown in Figure 8. We consider using four reference nodes in a 2D environment; this selection assumes that each reference node will be located in each corner of the rectangular shape measurement area. Thus, the interpolation techniques will likely have a better linear relationship between each reference node and its corresponding distance. We have applied more reference nodes for fingerprint-based in the same measurement area and can be found in [50-52]. Some results suggested that more reference nodes yield better accuracy (scalability). However, our previous publications do not consider interpolation techniques.

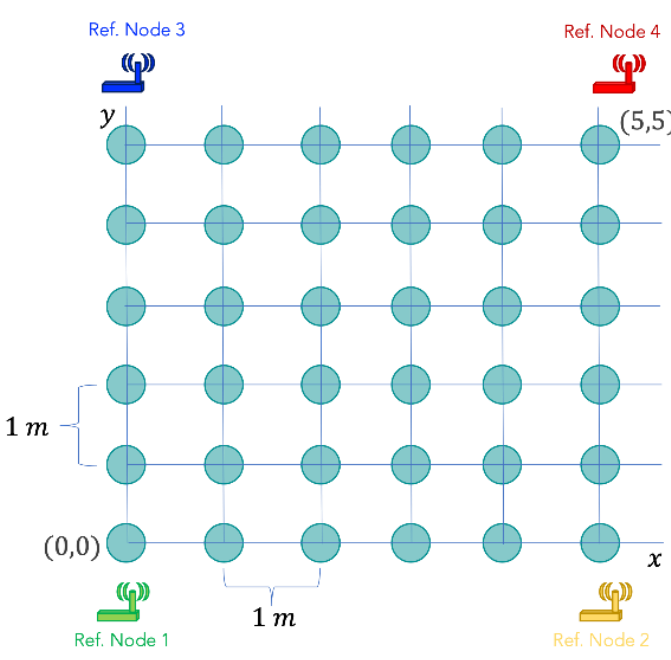

(a)

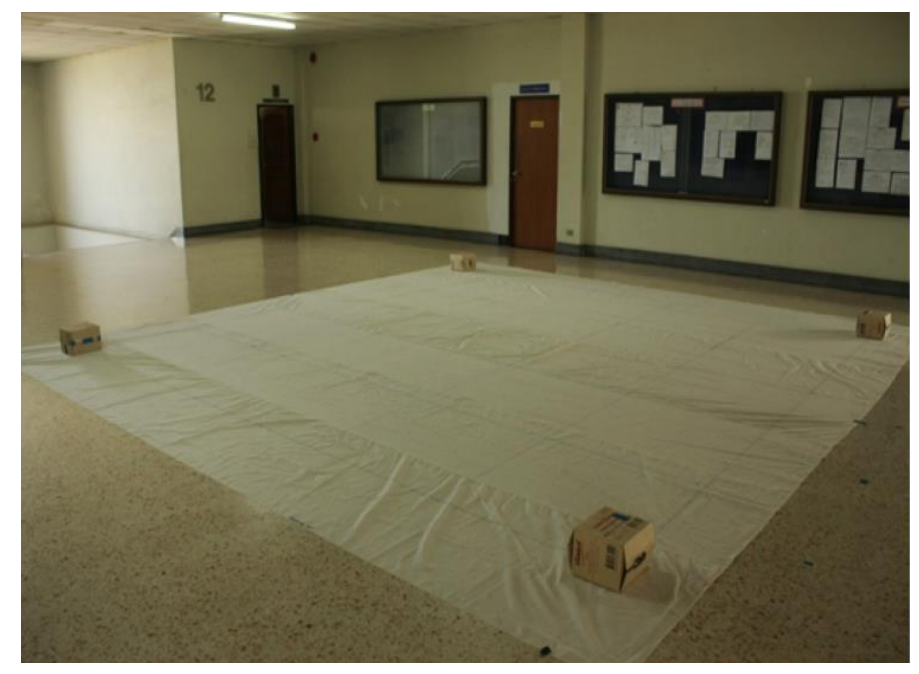

(b)

Figure 8. 2D area of interest measurement campaign, a) layout illustration, and b) actual setup.

The four reference nodes are deployed in each rectangular shape's corner, as depicted in Figure 8 . The measurement grid is $1 \times 1 \mathrm{~m}^{2}$; we have 36 fingerprint locations measurement for the database construction in $5 \times 5 \mathrm{~m}^{2}$ area of interest. The measurement area size has been validated since the RSSI values from the XBee-24ZB device are best in under 8$9 \mathrm{~m}$. The $5 \times 5 \mathrm{~m}^{2}$ is still relatively line-of-sight for the lobby itself to support our approach to ensure that the relationship between signal strength and distance is log-loss or linearly decreased. The interpolation techniques need the linear relationship, which is the distance vs. signal strength. ZigBee standard works in the center frequency of $2.4 \mathrm{GHz}$, the same band with Wi-Fi. To minimize the interference between signals, we turned off all Wi-Fi access points in the measurement area while doing the measurement. The target node is statically placed inside the area of interest. Some positions are the same positions with the fingerprint locations to ensure the quality of the database, some others between two or in the center of four fingerprint locations. The detail of target locations and target type position is shown in Figure 9 .

The diagonally, vertically, and horizontally placement are considered because of the placement of four reference nodes shown in Figure 8a. The effects of the distance and orientation of the target with the reference nodes can be explored by these target positions setup. 


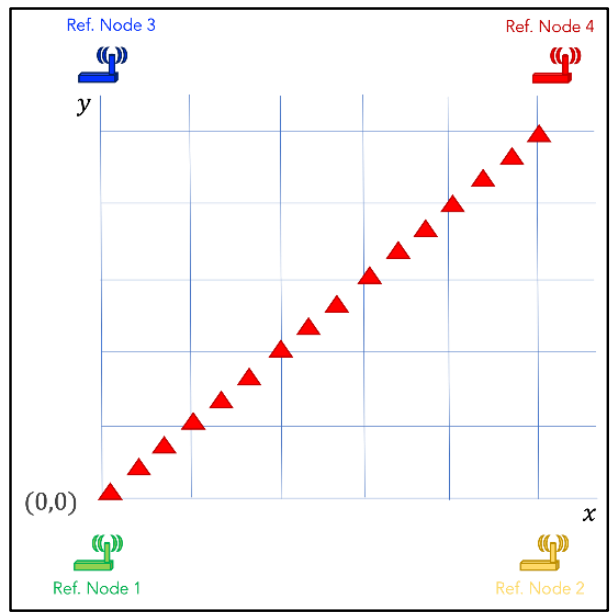

(a)

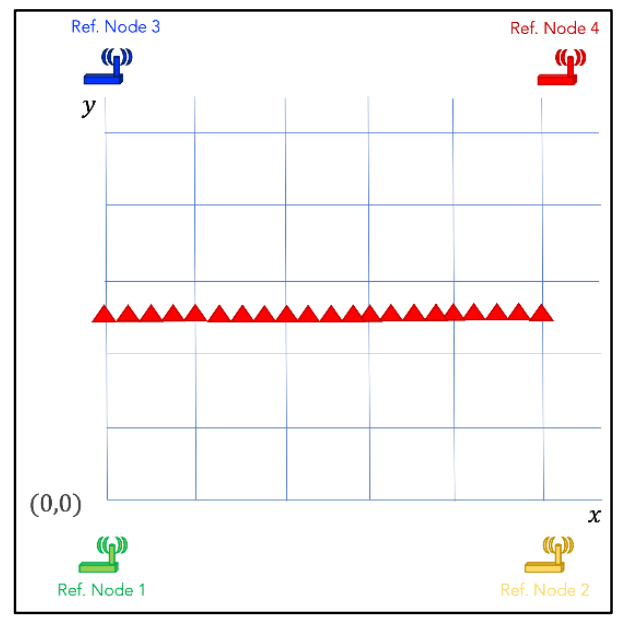

(c)

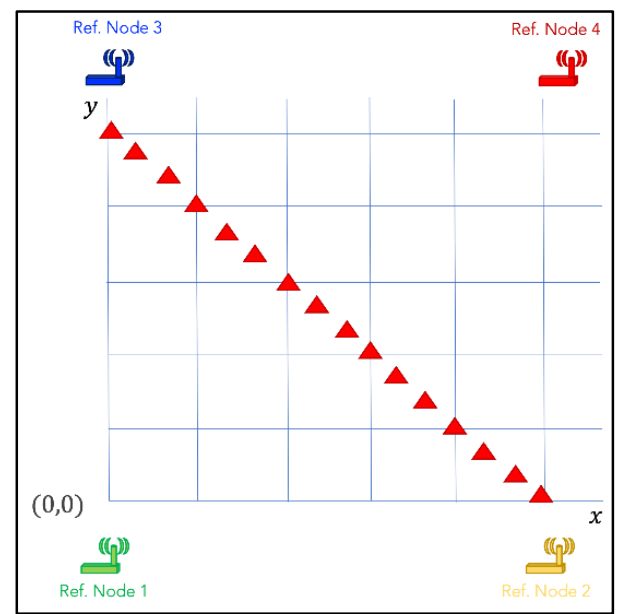

(b)

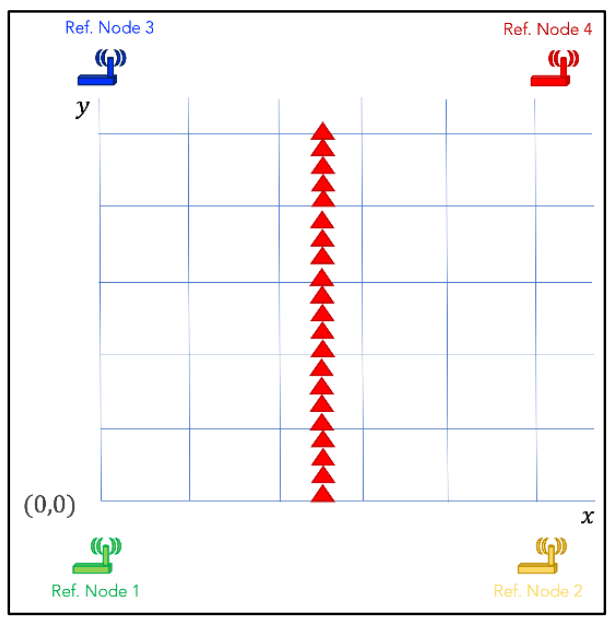

(d)

Figure 9. Target types' position, a) type 1, b) type 2, c) type 3, d) type 4.

\section{The 3D Measurement Campaign}

To simulate the multi-story building with different levels/floors, we used a four-level wooden bookshelf. We also considered three scenarios in this 3D environment; clean environment, human body effects, and interference objects of books. The body effect is considered by a standing person in $1 \mathrm{~m}$ distance from the bookshelf. The bookshelf's dimensions are $92 \times 25 \times 152 \mathrm{~cm}^{3}$ and the detailed dimension and illustration are depicted in Figure 10 .

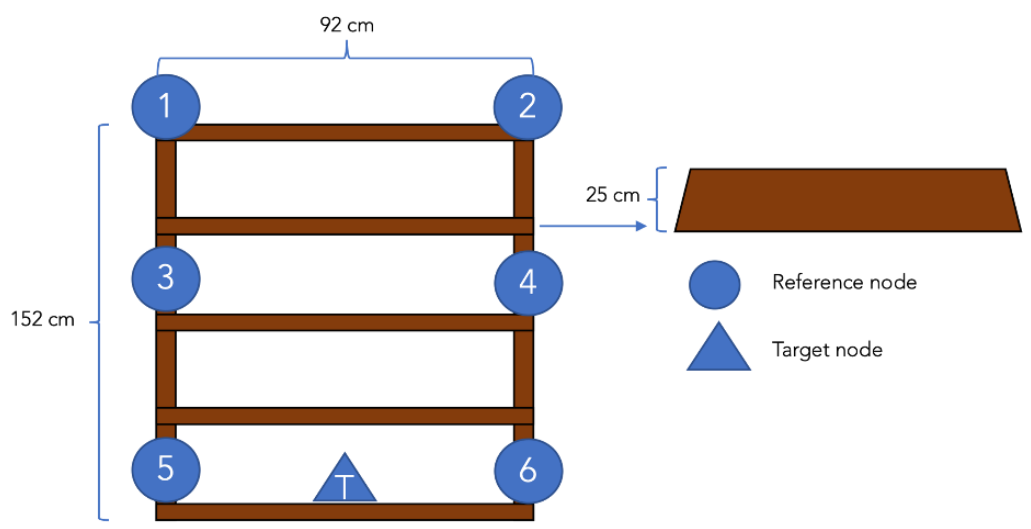

Figure 10. The illustration of a 3D environment in a bookshelf.

The fingerprint node (FP node) and target node can be illustrated in Figure 11. Unlike in the 2D environment, in the 3D environment, we employed six reference nodes. This selection ensures that the elevation properties are well represented as the XBee antenna directional pattern is more horizontally shaped. Moreover, we have only six reference nodes in the dataset's measurement setup and have not yet published the approach. The target, $T$ is placed in the different levels of the bookshelf statically. 


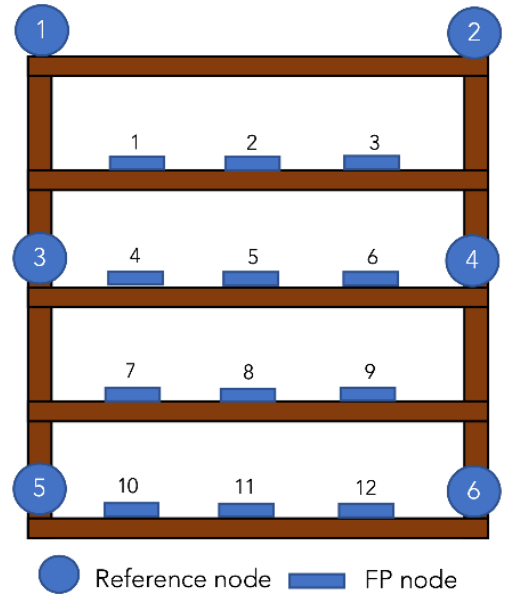

(a)

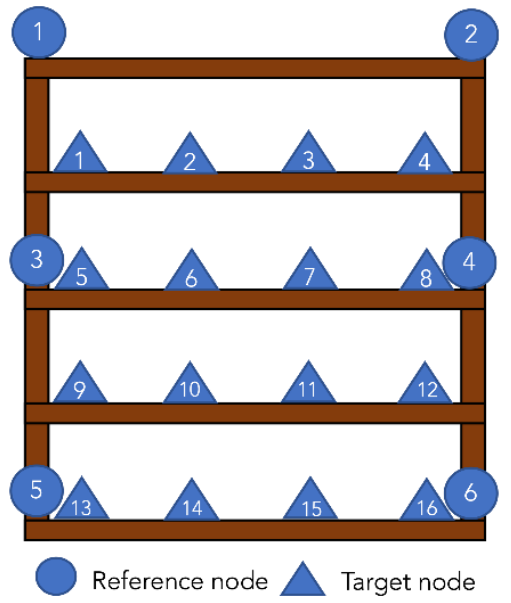

(b)

Figure 11. The illustration of a 3D environment in a bookshelf, a) fingerprint position and b) target position.
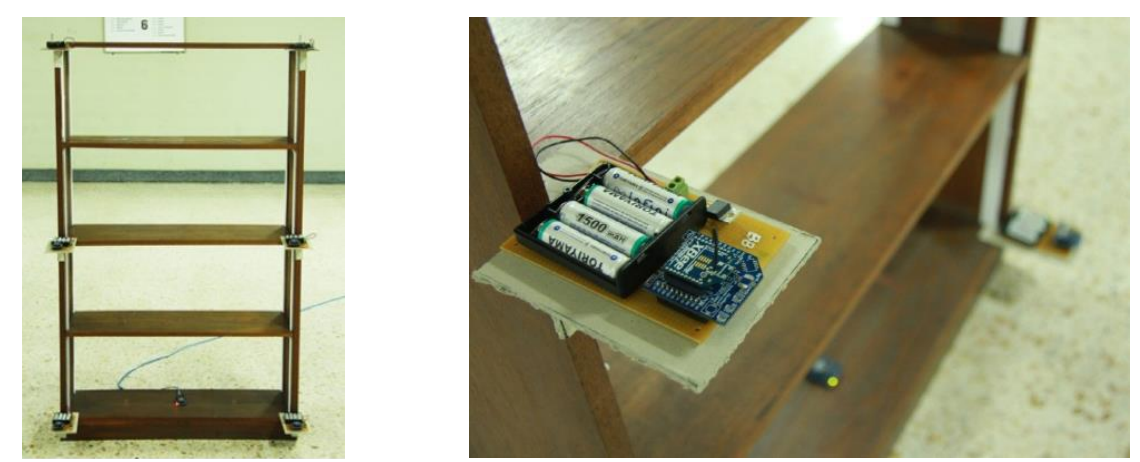

Figure 12. The real setup of $3 D$ environment in a bookshelf.

The 12 FP node locations are used to collect the RSSI values for the fingerprint database. Similar to the 2D environment, here, we also use XBee-24ZB as the core of the system. The fingerprint grid size is $22 \times 12.5 \times 35 \mathrm{~cm}^{3}$, for length, width, and elevation. The total target node position is 16, located between the FP nodes or near the reference nodes. Figure 12 shows the actual placement of XBee-24ZB for the 3D environment setup. The coordinate of fingerprint and target position in the $3 \mathrm{D}$ environment is presented in Table 4.

Table 3. 3D fingerprint and target coordinate.

\begin{tabular}{|c|c|c|c|}
\hline Fingerprint Node & Coordinate $(x, y, z)$ & Target Node & Coordinate $(x, y, z)$ \\
\hline 1. & $22 \mathrm{~cm}, 12.5 \mathrm{~cm}, 105 \mathrm{~cm}$ & 1. & $11 \mathrm{~cm}, 12.5 \mathrm{~cm}, 105 \mathrm{~cm}$ \\
\hline 2. & $44 \mathrm{~cm}, 12.5 \mathrm{~cm}, 105 \mathrm{~cm}$ & 2. & $33 \mathrm{~cm}, 12.5 \mathrm{~cm}, 105 \mathrm{~cm}$ \\
\hline 3. & $66 \mathrm{~cm}, 12.5 \mathrm{~cm}, 105 \mathrm{~cm}$ & 3. & $55 \mathrm{~cm}, 12.5 \mathrm{~cm}, 105 \mathrm{~cm}$ \\
\hline 4. & $22 \mathrm{~cm}, 12.5 \mathrm{~cm}, 70 \mathrm{~cm}$ & 4. & $77 \mathrm{~cm}, 12.5 \mathrm{~cm}, 150 \mathrm{~cm}$ \\
\hline 5. & $44 \mathrm{~cm}, 12.5 \mathrm{~cm}, 70 \mathrm{~cm}$ & 5. & $11 \mathrm{~cm}, 12.5 \mathrm{~cm}, 70 \mathrm{~cm}$ \\
\hline 6. & $66 \mathrm{~cm}, 12.5 \mathrm{~cm}, 70 \mathrm{~cm}$ & 6. & $33 \mathrm{~cm}, 12.5 \mathrm{~cm}, 70 \mathrm{~cm}$ \\
\hline 7. & $22 \mathrm{~cm}, 12.5 \mathrm{~cm}, 35 \mathrm{~cm}$ & 7. & $55 \mathrm{~cm}, 12.5 \mathrm{~cm}, 70 \mathrm{~cm}$ \\
\hline 8. & $44 \mathrm{~cm}, 12.5 \mathrm{~cm}, 35 \mathrm{~cm}$ & 8. & $77 \mathrm{~cm}, 12.5 \mathrm{~cm}, 70 \mathrm{~cm}$ \\
\hline 9. & $66 \mathrm{~cm}, 12.5 \mathrm{~cm}, 35 \mathrm{~cm}$ & 9. & $11 \mathrm{~cm}, 12.5 \mathrm{~cm}, 35 \mathrm{~cm}$ \\
\hline 10. & $22 \mathrm{~cm}, 12.5 \mathrm{~cm}, 0 \mathrm{~cm}$ & 10. & $33 \mathrm{~cm}, 12.5 \mathrm{~cm}, 35 \mathrm{~cm}$ \\
\hline 11. & $44 \mathrm{~cm}, 12.5 \mathrm{~cm}, 0 \mathrm{~cm}$ & 11. & $55 \mathrm{~cm}, 12.5 \mathrm{~cm}, 35 \mathrm{~cm}$ \\
\hline 12. & $66 \mathrm{~cm}, 12.5 \mathrm{~cm}, 0 \mathrm{~cm}$ & 12. & $77 \mathrm{~cm}, 12.5 \mathrm{~cm}, 35 \mathrm{~cm}$ \\
\hline- & - & 13. & $11 \mathrm{~cm}, 12.5 \mathrm{~cm}, 0 \mathrm{~cm}$ \\
\hline- & - & 14. & $33 \mathrm{~cm}, 12.5 \mathrm{~cm}, 0 \mathrm{~cm}$ \\
\hline- & - & 15. & $55 \mathrm{~cm}, 12.5 \mathrm{~cm}, 0 \mathrm{~cm}$ \\
\hline- & - & 16. & $77 \mathrm{~cm}, 12.5 \mathrm{~cm}, 0 \mathrm{~cm}$ \\
\hline
\end{tabular}




\section{3-6-Database Enhancement}

We offer database enhancement by constructing a synthetic database to tackle sparsity.

\section{The 2D Measurement Database Enhancement}

Some data at measured RSSI database or we called as fingerprint are omitted and replaced with artificial data according to Figure 13. The blue dots represent the measurement data, and the point other than the blue dots are the points where the data was replaced. From the blue dots, we created the synthetic data. The red dots are reproduced from interpolation or regression using the blue dots on the x-axis. While green dots were created using blue dots on the yaxis. Last step, artificial data in orange dots were made using the green ones. Later, we order these artificial data as bottom to top rows, left to right columns, e.g., the first red dot in the bottom grid is in the first order $(1,0)$, continue to the second red dot as $(4,0)$. Move to the upper row; the green dot is in order three as $(0,1)$, then continue to order 20 as a red dot in (4,5). The artificial data order and coordinate can be summarized in Table 3 . In the next step, we added artificial data into the measurement database to have a new database with a $0.5 \times 0.5 \mathrm{~m}^{2}$ grid database from the fingerprint database that we created using bilinear interpolation, polynomial regression, and polynomial interpolation and named as $f p+i n t B i l \_d b, f p+$ regPoly, and $f p+i n t P o l y \_d b$. The method to make the database denser is applied based on the authors' works in $[53,54]$.

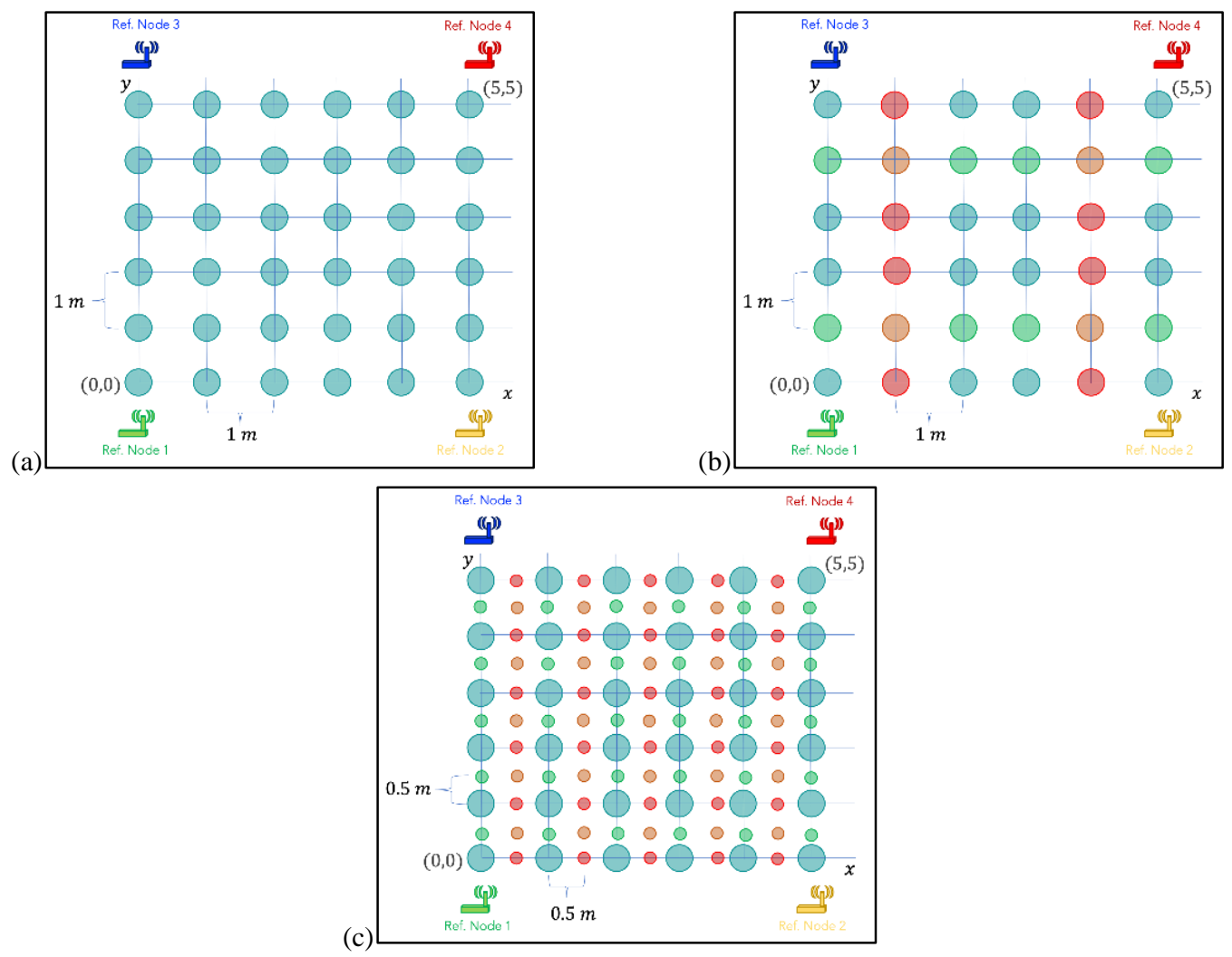

Figure 13. Database: a) Actual, b) Replacement scheme in $1 \times 1 \mathrm{~m}^{2}$, c) Synthetic database for $0.5 \times 0.5 \mathrm{~m}^{2}$.

Table 4. Data Replacement Order.

\begin{tabular}{cccccc}
\hline \multirow{2}{*}{ Order No. } & \multicolumn{2}{c}{ Coordinate } & & \multicolumn{2}{c}{ Coordinate } \\
\cline { 2 - 3 } \cline { 5 - 6 } & $\boldsymbol{x}$ & $\boldsymbol{y}$ & & $\boldsymbol{x}$ & $\boldsymbol{y}$ \\
\hline 1. & 1 & 0 & 11. & 1 & 3 \\
2. & 4 & 0 & 12. & 4 & 3 \\
3. & 0 & 1 & 13. & 0 & 4 \\
4. & 1 & 1 & 14. & 1 & 4 \\
5. & 2 & 1 & 15. & 2 & 4 \\
6. & 3 & 1 & 16. & 3 & 4 \\
7. & 4 & 1 & 17. & 4 & 4 \\
8. & 5 & 1 & 18. & 5 & 4 \\
9. & 1 & 2 & 19. & 1 & 5 \\
10. & 4 & 2 & 20. & 4 & 5 \\
\hline
\end{tabular}




\section{The 3D Measurement Database Enhancement}

The 3D measurement database enhancement was applying interpolation and regression to generate artificial data between or within the measured data range; we also created new data outside the range or what we call extrapolation in positions $1,4,5,8,9,12,13,16$ according to Figure $11 \mathrm{~b}$.

\section{4- Results and Discussions}

\section{4-1-2D Environment}

We present the results by using Average Distance Error (ADE) comparison of bilinear interpolation, polynomial interpolation, and polynomial regression for replacement and enhancement data schemes applied as fingerprint databases.

\section{RSSI Data Replacement}

We examined the discrepancy values between prediction results and the actual RSSI measurements for all reference nodes (four). This RSSI discrepancy is only observed in the replacement order in Table 3. We evaluated the RSSI values predicted results; data is presented in the form of the difference between the data created using polynomial interpolation, polynomial regression, and bilinear interpolation on the RSSI value of the measurement results. Figure 15 showed that the highest error value is at order no. 14, with an error from the polynomial interpolation is $5.97 \mathrm{dBm}$. This result shows a high difference compared to the error from polynomial regression and bilinear interpolation, with $2.44 \mathrm{dBm}$ and 2.20 $\mathrm{dBm}$, respectively. This result is also the highest among all RSSI prediction error data from reference node 1 . While the slightest error is generated at point 11 using the polynomial interpolation method with an error value of $0.00 \mathrm{dBm}$.

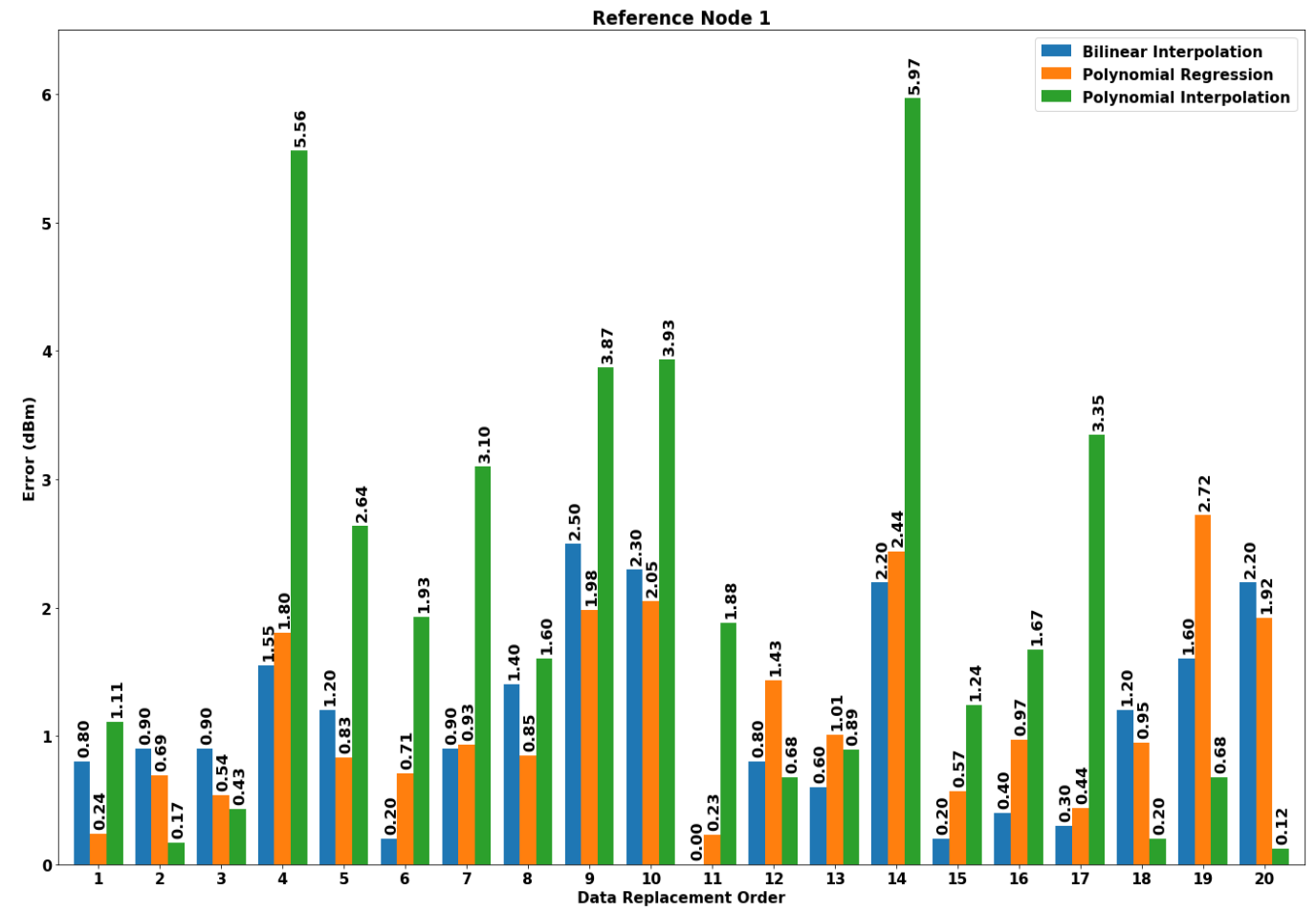

Figure 15. RSSI values error for reference node 1.

Figure 16 shows the RSSI heatmap comparison for reference node 1. It shows the gradation of the RSSI values as the brighter color refers to the highest RSSI, and the darker color is the low RSSI values. The average RSSI means from the five-time measurement we took the mean value. For interpolation and regression, it was applied to each measurement dataset and also took the average values. Figure 16 also shows the similarity pattern between measured RSSI and bilinear interpolation results. On the other hand, polynomial interpolation results are more scattered than the measurement. The results also confirmed the RSSI error discrepancy that is relatively high for polynomial interpolation, as observed in Figure 15. 


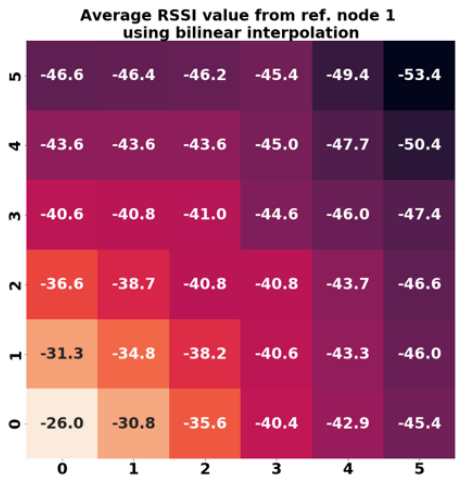

(a)

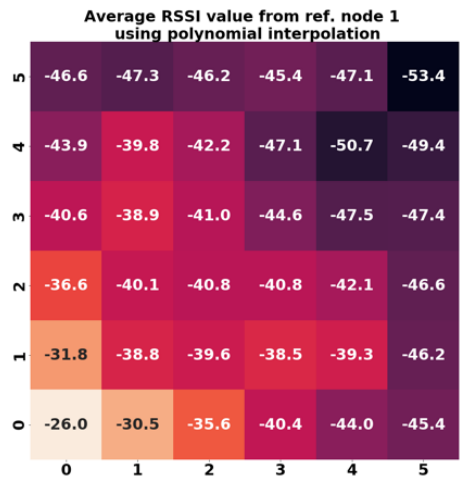

(c)

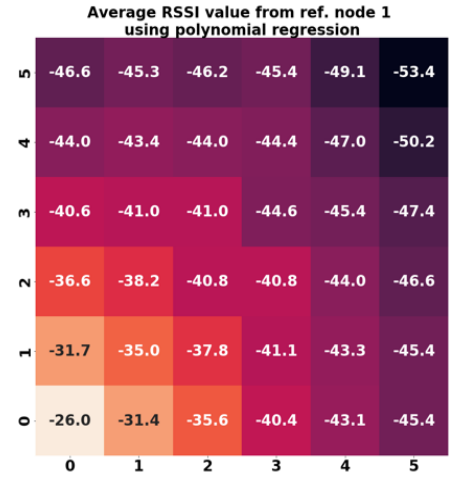

(b)

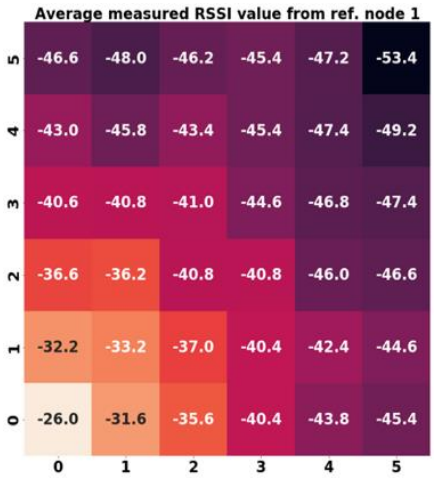

(d)

Figure 16. RSSI heatmap reference node 1: a) Bilinear inter., b) Polynomial reg., c) Polynomial inter., d) Measurement.

Figure 17 shows the RSSI values discrepancy for reference node 2. It shows that the majority of the RSSI values prediction using the polynomial interpolation produces a more significant error than polynomial regression and bilinear interpolation. The most significant prediction errors are at orders 11, 14, and 15, with $3.27 \mathrm{dBm}, 3.83 \mathrm{dBm}$, and 4.44 $\mathrm{dBm}$. On the other hand, the polynomial regression method produces the most significant errors at orders 9, 12, and 19, with each point having errors of $2.38 \mathrm{dBm}, 2.73 \mathrm{dBm}$, and $3.14 \mathrm{dBm}$, respectively. At the same time, the most negligible error results in the prediction of RSSI values using polynomial interpolation, polynomial regression, and bilinear interpolation are $0.09 \mathrm{dBm}, 0.02 \mathrm{dBm}$, and $0.10 \mathrm{dBm}$. Figure 18 depicts the RSSI heatmap comparison for reference node 2 . The brighter colour gradation is origin from the bottom right as the reference node two positions in the measurement.

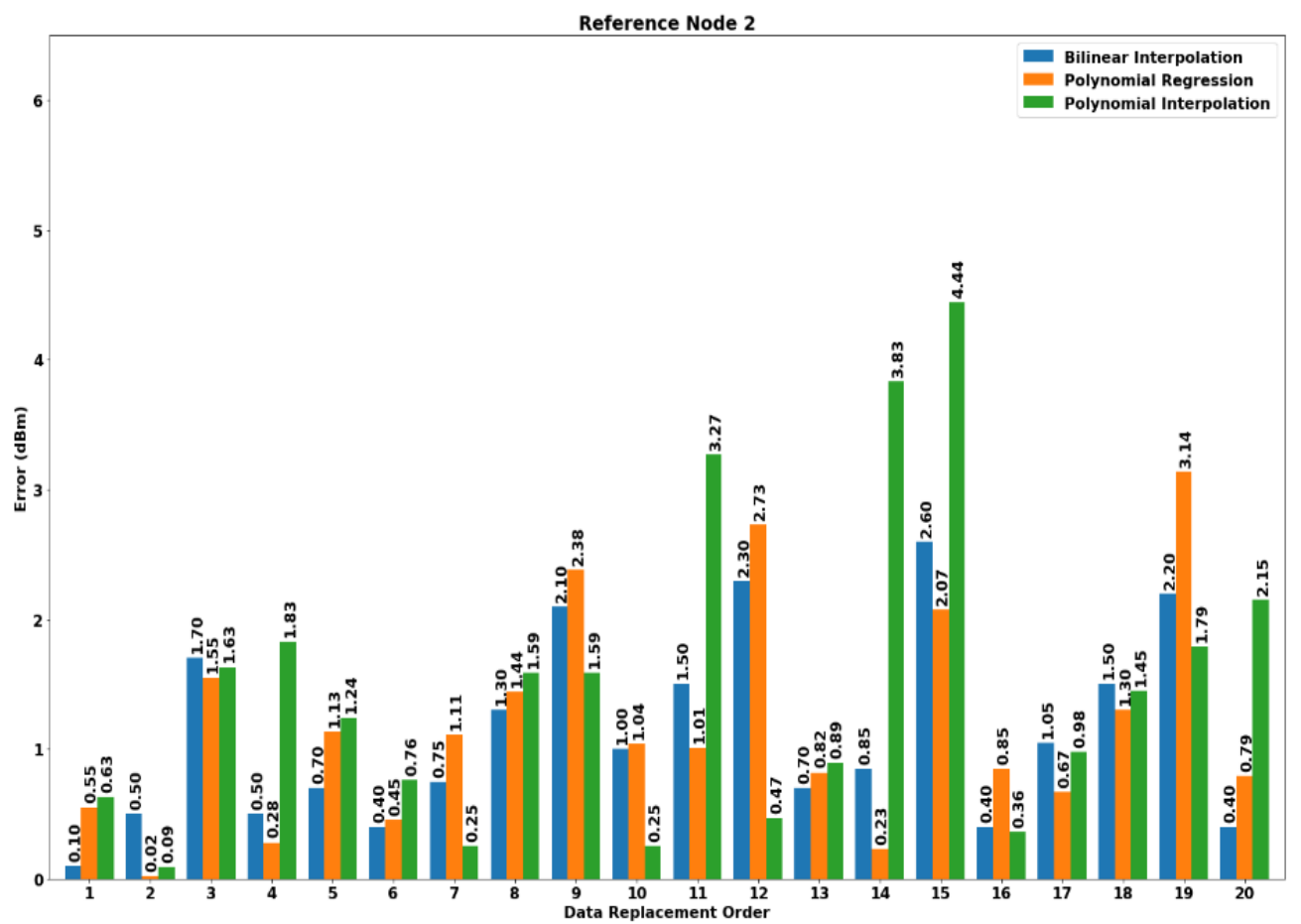

Figure 17. RSSI values error for reference node 2 


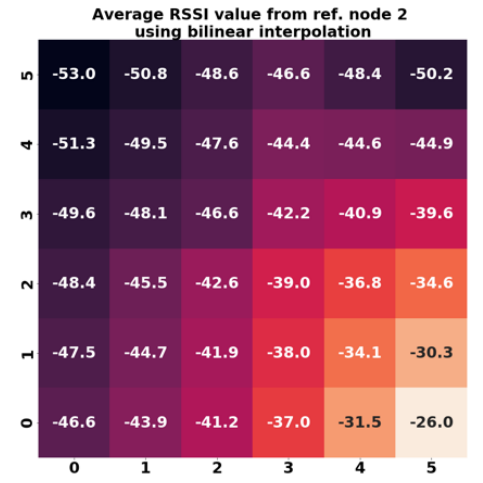

(a)

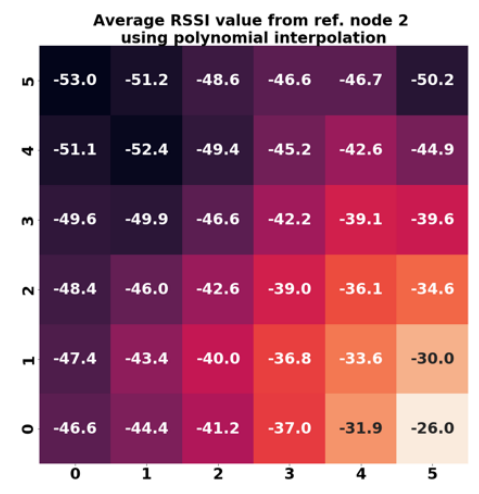

(c)

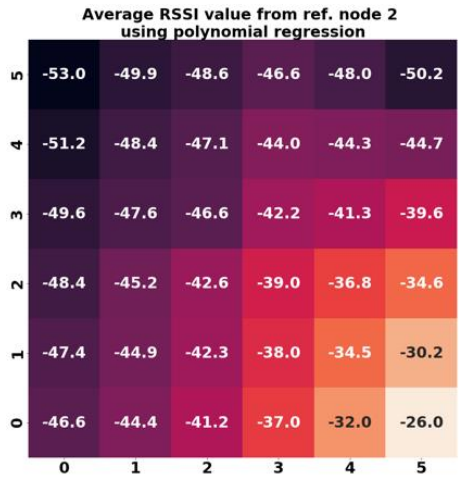

(b)

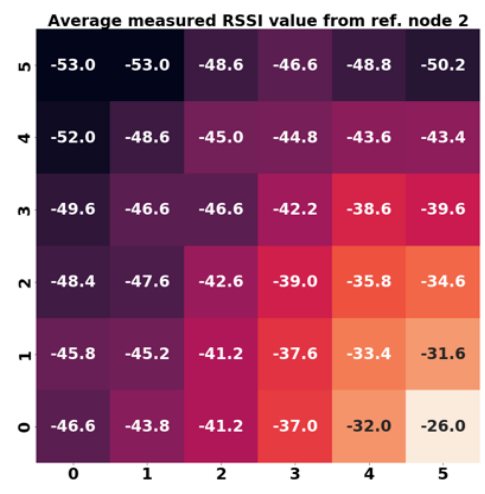

(d)

Figure 18. RSSI heatmap reference node 2. a) Bilinear inter., b) Polynomial reg., c) Polynomial inter., d) Measurement

Figure 19 shows the RSSI error values discrepancy comparison between the three proposed methods and actual RSSI values from measurement. We found that the most significant errors are at orders 2,8 , and 18, where the replacement of RSSI values using the polynomial interpolation method produces errors of $3.89 \mathrm{dBm}, 4.53 \mathrm{dBm}$, and $3.47 \mathrm{dBm}$. In reference node 3 , the minor RSSI error from polynomial interpolation, polynomial regression, and bilinear interpolation are $0.06 \mathrm{dBm}, 0.08 \mathrm{dBm}$, and $0.10 \mathrm{dBm}$. Overall, the mean RSSI prediction error using polynomial interpolation, polynomial regression, and bilinear interpolation methods are $1.81 \mathrm{dBm}, 0.95 \mathrm{dBm}$, and $0.81 \mathrm{dBm}$, respectively. To observe the precise RSSI values distribution, Figure 20 shows the RSSI heatmap comparison for reference node three, and the brighter to darker color gradation originates from the upper left (the position of reference node 3 in measurement).

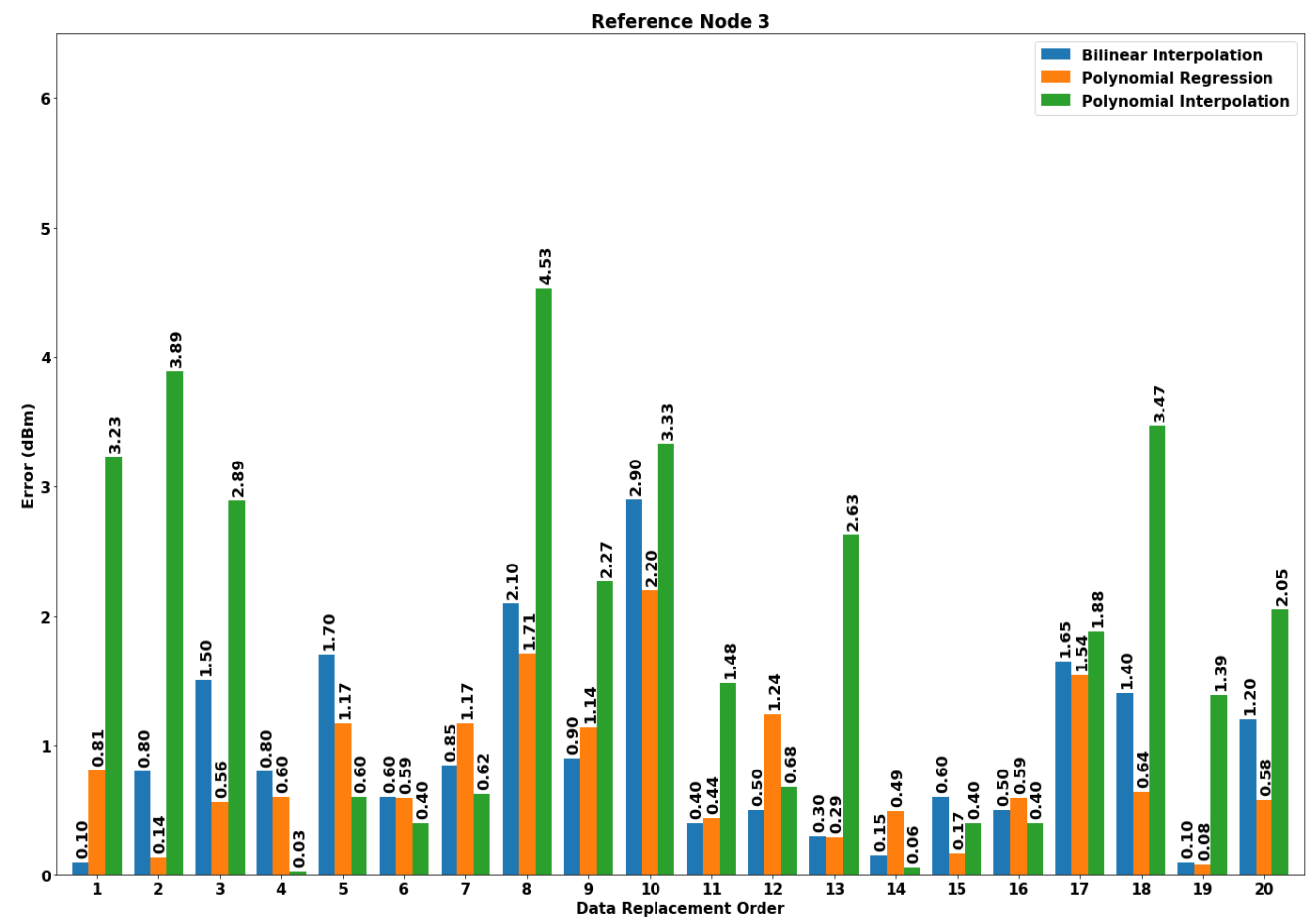

Figure 19. RSSI values error for reference node 3 


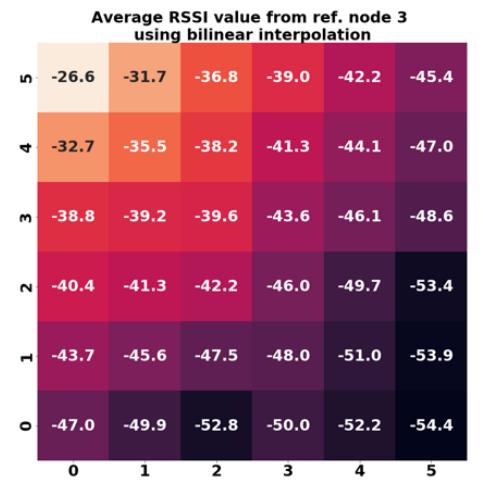

(a)

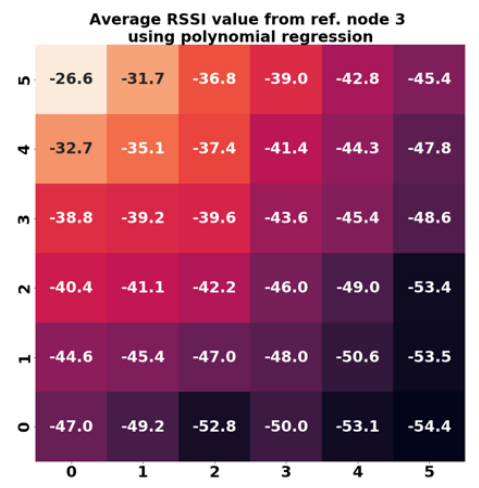

(c)

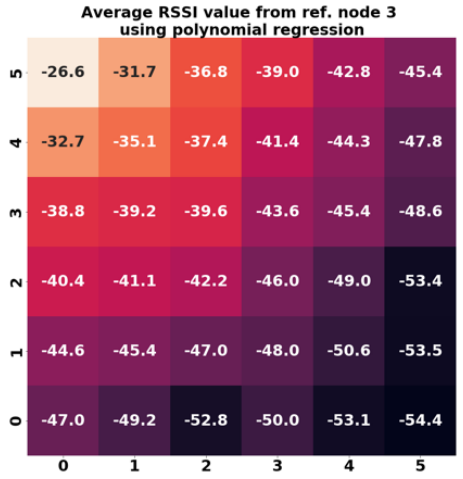

(b)

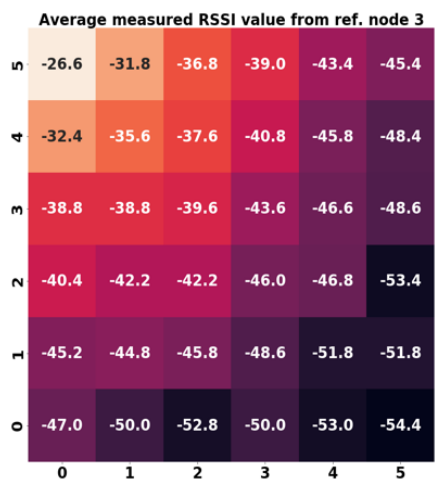

(d)

Figure 20. RSSI heatmap reference node 3. a) Bilinear inter., b) Polynomial reg., c) Polynomial inter., d) Measurement

The RSSI values discrepancy from three methods compared to the measurement for the reference node four is shown in Figure 21. The results also show that polynomial interpolation produces the most significant error with the largest error at the first order with an error value of $5.11 \mathrm{dBm}$. In comparison, the polynomial regression method and bilinear interpolation at the same point resulted in an error of $2.92 \mathrm{dBm}$ and $3.90 \mathrm{dBm}$, respectively. By implementing polynomial interpolation, polynomial regression, and bilinear interpolation methods, the mean RSSI prediction error is $1.74 \mathrm{dBm}, 1.16 \mathrm{dBm}$, and $1.04 \mathrm{dBm}$, respectively. From these results, we can conclude that the bilinear interpolation performed relatively better than polynomial interpolation and regression. Figure 22 shows the RSSI heatmap comparison for reference node 4 .

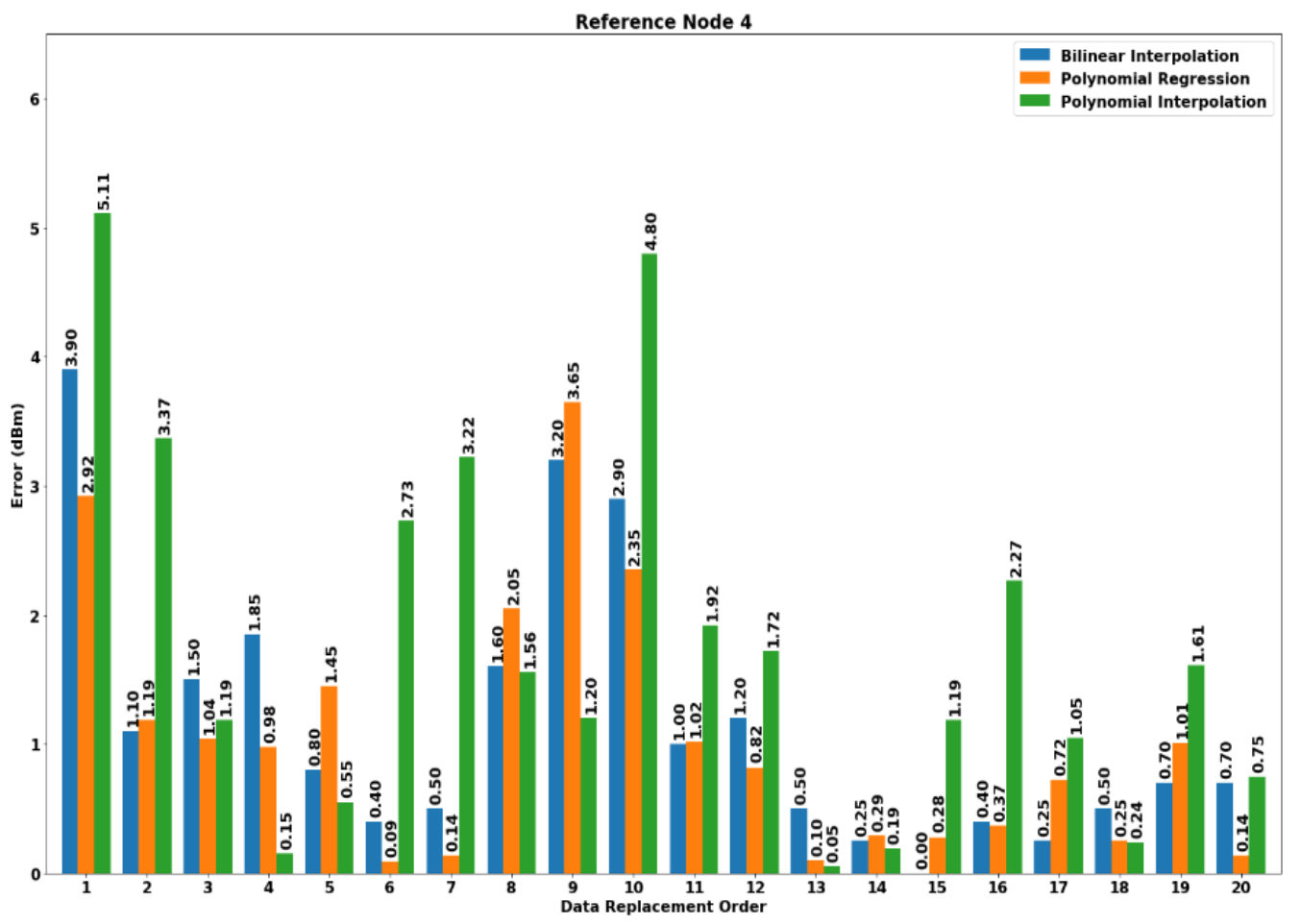

Figure 21. RSSI values error for reference node 4 
(a)

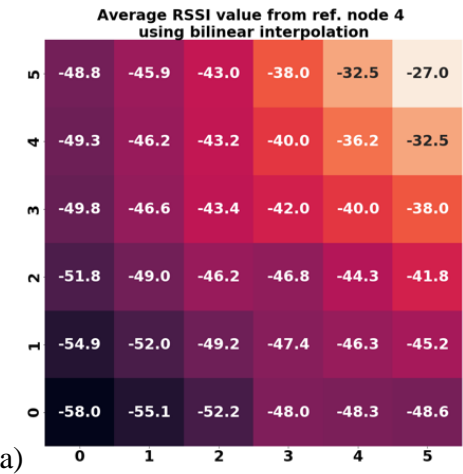

Average RSSI value from ref. node 4

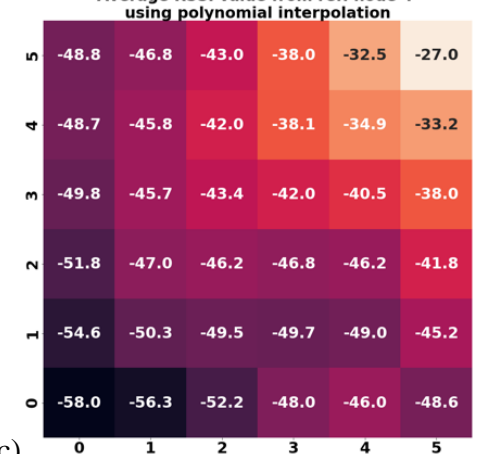

(b)
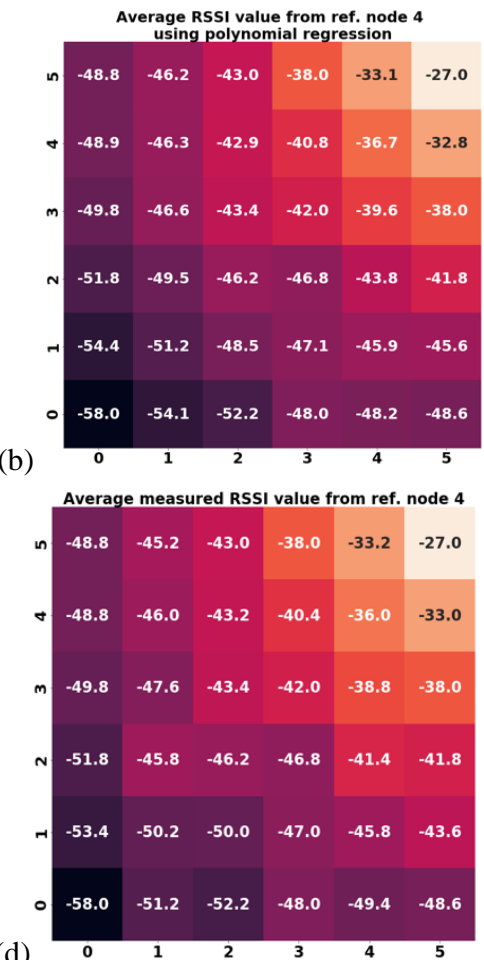

(d)

$\begin{array}{llllll}0 & 1 & 2 & 3 & 4 & 5\end{array}$

Figure 22. RSSI heatmap reference node 4. a) Bilinear inter., b) Polynomial reg., c) Polynomial inter., d) Measurement

\section{Localization Results}

The synthetic fingerprint databases are labelled as and fp+intBil_db, fp+regPoly_db, fp+intPoly_db, for bilinear interpolation, regression polynomial, and polynomial interpolation, respectively. For $2 \mathrm{D}$ environment, we consider the 4 types of target position namely as target type 1-4. We compare the localization results, fp+intBil_db, fp+regPoly_db, fp+intPoly_db databases with the actual measured fingerprint database, fp_meas_db. For the target types 1-4, the comparison of ADE results can be seen in Figure 23. The database used in the positioning results is illustrated in Figure 14. By implementing polynomial interpolation, polynomial regression, and bilinear interpolation methods, new data is added so that a database is formed with the amount of data at points where the RSSI value is not measured. The new database is then labelled as fp+intPoly_db, fp+regPoly_db, and fp+intBil_db. The three databases are then compared with the fp_meas_db database.
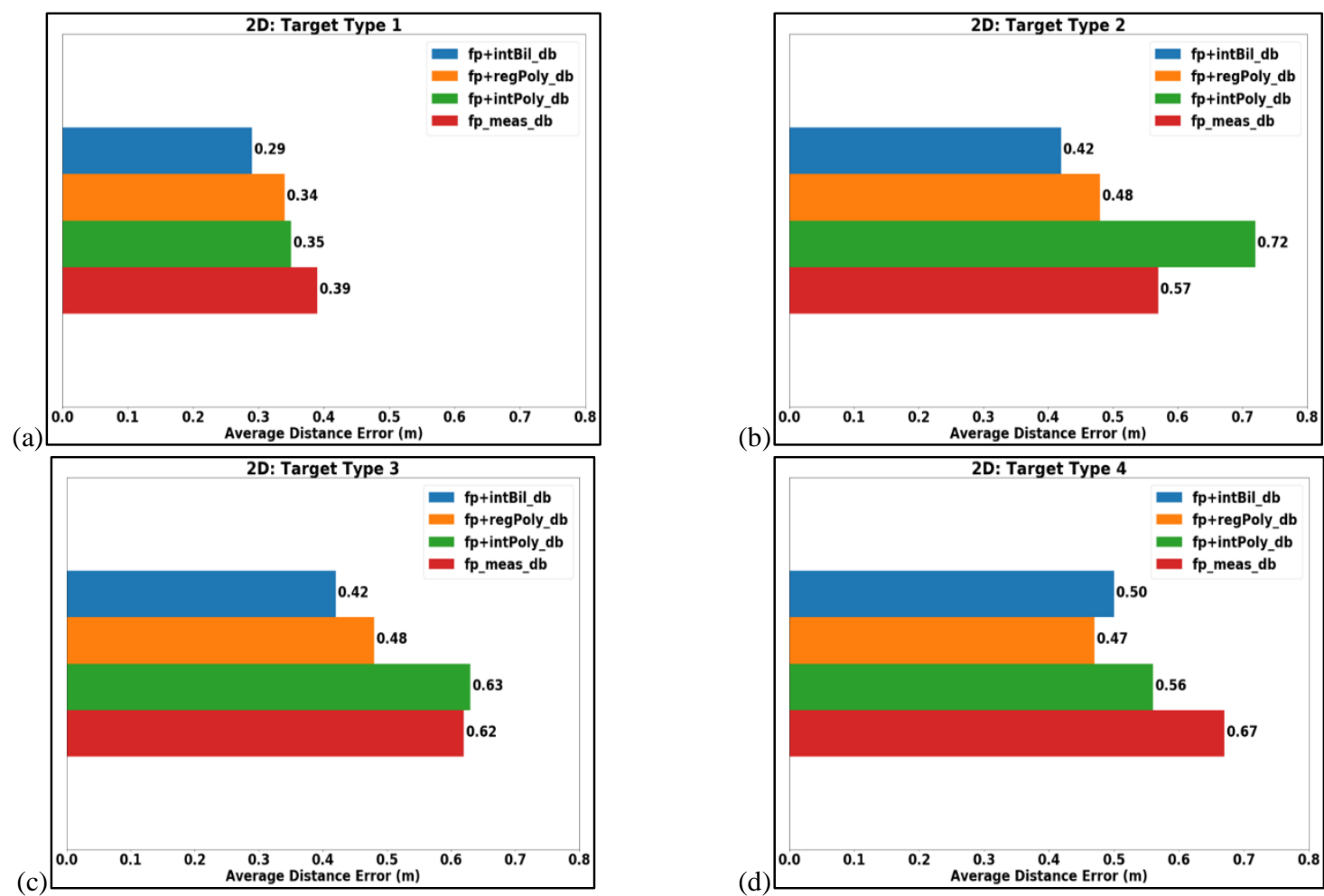

Figure 23. Localization results comparison, a) target type 1, b) target type 2, c) target type 3, and d) target type 4. 
For target type 1 in Figure 23.a)., the ADE values obtained using fp+intBil_db, fp+regPoly_db, fp+intPoly_db, and fp_meas_db are $0.29 \mathrm{~m}, 0.34 \mathrm{~m}, 0.35 \mathrm{~m}$ and 0.39 , respectively. These results show that applying all the interpolation and regression techniques can reduce the average error of $0.1 \mathrm{~m}$ in the target type 1 scenario. The database scarcity, or in other words, by reducing the number of database size in the offline database and we compensated with the classical interpolation and regression technique, has been proven to improve the localization performance.

For target type 2, the ADE values obtained using fp+intBil_db, fp+regPoly_db,fp+intPoly_db, and fp_meas_db are $0.42,0.48,0.72$, and $0.57 \mathrm{~m}$ as shown in Figure 23.b). Positioning results with reduced lattice size database using polynomial interpolation method resulted in higher ADE values with an increase of $0.15 \mathrm{~m}$. Meanwhile, polynomial regression and bilinear interpolation reduced ADE by 0.09 and $0.15 \mathrm{~m}$, respectively.

For the horizontal movement of target type 3, the positioning results using fp+intBil_db, fp+regPoly_db, $\mathrm{fp}+$ intPoly_db, and fp_meas_db is shown in Figure 23.c). Only fp+intPoly_db obtained the less accurate results compared to fp+regPoly_db, and fp+intBil_db. The $0.2 \mathrm{~m}$ error improvement was achieved by the fp+intBil_db, which so far achieved the best results. The last scenario is the target type 4 in the vertical movement. Figure 23.d) shows the results of improvement by all types of the additional database; fp+intBil_db, fp+regPoly_db, and fp+intPoly_db. These results proved that the interpolation and regression for enhancing the database by adding the artificial points between actual measurement points were successfully implemented and validated.

We would like to explore how the RSSI values from 4 reference nodes are placed in each corner of the rectangular shape measurement area by selecting these four target position types. In target types 1 and 2 , the diagonal is easier to imagine that the gradation of the RSSI values will follow the diagonal line. However, as the position is somehow in the middle of the area of interest for horizontal and vertical target type positions, it is expected to make the estimation more prone to error, as observed in Figures 23.c and 23.d.

\section{4-2-3D Environment}

Unlike 2D environment, results for the 3D environment were analyzed and validated by considering three types of scenarios in the bookshelf; the first scenario is a clean environment when there is no obstruction objects on the bookshelf, the second scenario, during the measurement campaign, the first author standing close to the bookshelf $(0.5 \mathrm{~m})$ facing front. The third scenario is to place books and other stationaries between the reference nodes in every level of the bookshelf having the reference nodes. We expected that by putting a kind of 'noise' in our measurement, we could observe the improvement when applying the interpolation and regression technique. Fingerprint databases generated from the replacement of measurement data are also labeled as fp+intBil_db, fp+regPoly_db, fp+intPoly_db, and fp_meas_db for bilinear interpolation, polynomial regression, polynomial interpolation, and actual fingerprint database, respectively. These techniques not only augment the data inside the data range but are also used to generate data outside the range (i.e., extrapolation). Figure 24 shows the results for three scenarios in the 3D environment.

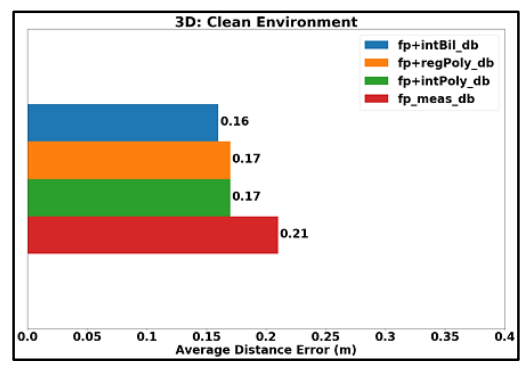

(a)

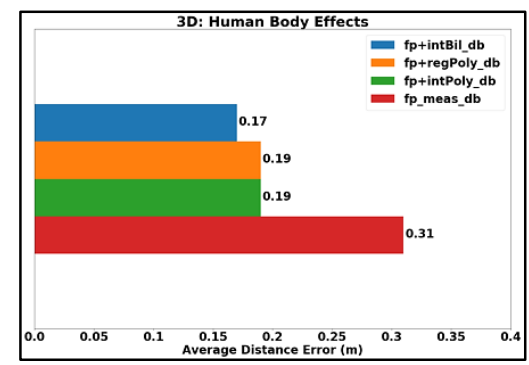

(b)

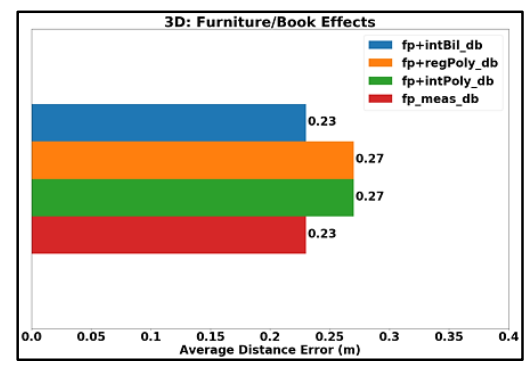

(c)

Figure 24. Localization results comparison, a) clean environment, b) human body effects, c) furniture/book effects

A clean environment can also be called ideal conditions that we set up without any obstacles in the area of interest. We observe that all enhancement methods can reduce ADE compared to actual fingerprints in this condition. Data quantity enhancement using bilinear interpolation has the most significant ADE reduction. Polynomial interpolation and regression have the same results as shown in Figure 24.a). In the human body effects scenario, we can observe that the localization results were less accurate than the clean environment.

For human body effects, an approach of interpolation applying Kriging for wireless body area network (WBAN) indoor localization has been proposed in [55]. Kriging is a mechanism to predict spatial information for estimating a value at a specific location using actual locations. The paper discussed the possibility of implementing Kriging interpolation to the constructed artificial database and found that by Kriged fingerprint, the accuracy is improved. In this paper, we did not analyze the propagation mechanism. However, we expect that a person standing near the bookshelf will likely affect the localization results. From Figure 24.b), there was a slightly added measured error for $3 \sim 8 \mathrm{~cm}$. Polynomial interpolation and regression achieved the same result, but they were less accurate than the actual measurement fingerprint data. Bilinear interpolation has a very tiny accuracy margin better than measurement fingerprint database. 
For the furniture effects scenario, all enhancement methods can reduce ADE almost twice than using measurement fingerprint data. In other case studies, positioning results using polynomial interpolation and regression gave the same results in furniture obstacles. The bilinear interpolation always gave the minor error position. The object's existence between reference nodes created shadowing effects that even the synthesis database cannot handle. From these results, we can conclude that in the $3 \mathrm{D}$ cases, the signal obstruction in the horizontal direction affected the localization results because of the random signal fluctuation. Table 5 compares the results between our proposal and other studies. We emphasized the interpolation technique, the environment, and the performance metric.

Table 5. Performance Comparison with Other studies.

\begin{tabular}{|c|c|c|c|c|c|}
\hline No. & $\begin{array}{c}\text { Indoor } \\
\text { Localization }\end{array}$ & $\begin{array}{l}\text { Parameter and } \\
\text { Technology }\end{array}$ & Techniques & Environment & Performance Metric \\
\hline 1. & $\begin{array}{l}\text { The proposed } \\
\text { method }\end{array}$ & RSSI, ZigBee & $\begin{array}{l}\text { Bilinear, polynomial and } \\
\text { polynomial regression }\end{array}$ & $\begin{array}{l}\text { Real environment: 2D } \\
\text { and 3D }\end{array}$ & $\begin{array}{l}\text { Interpolation reduces estimated error } \\
\text { up to } 0.2 \mathrm{~m} \text { for } 2 \mathrm{D} \text { and } 0.13 \mathrm{~m} \text { for } 3 \mathrm{D} \text {. }\end{array}$ \\
\hline 2. & $\begin{array}{c}\text { Jingxue Bi, et.al. } \\
\text { [33] }\end{array}$ & RSSI, Wi-Fi & $\begin{array}{l}\text { Crowdsourcing and } \\
\text { interpolation }\end{array}$ & $\begin{array}{l}\text { Real environment: total } \\
3200 \mathrm{~m}^{2} \text { area. }\end{array}$ & $\begin{array}{l}\text { Improving the accuracy results up to } \\
20 \% \text { manual fingerprint, by using only } \\
25 \% \text { reference points. }\end{array}$ \\
\hline 3. & $\begin{array}{c}\text { Yanwei Li, et.al. } \\
\text { [32] }\end{array}$ & RSSI, Wi-Fi & $\begin{array}{c}\text { Classic narrowband path } \\
\text { loss model }\end{array}$ & Dataset from [56] & $\begin{array}{l}\text { Refined map with size } 0.75 \times 0.75 \mathrm{~m}^{2} \\
\text { (grid) improve accuracy up to } 63 \% \\
\text { from } 3.6164 \mathrm{~m} \text { to } 1.3294 \mathrm{~m}\end{array}$ \\
\hline 4. & $\begin{array}{l}\text { Julie Yixuan } \\
\text { Zhu, et.al. [34] }\end{array}$ & RSSI, Wi-Fi & $\begin{array}{l}\text { Spatio-temporal }(\mathrm{S}-\mathrm{T}) \\
\text { similarity model which }\end{array}$ & $\begin{array}{l}\text { Real environment, area } \\
\text { of } 36 \times 18 \mathrm{~m}^{2}\end{array}$ & $\begin{array}{l}\text { By using the proposed method, the } \\
\text { improvement in interpolation and } \\
\text { positioning accuracy up to } 7 \% \text { and } \\
32 \% \text {, respectively. }\end{array}$ \\
\hline 5. & $\begin{array}{l}\text { Yongliang Sun, } \\
\text { et.al. [35] }\end{array}$ & RSSI, Wi-Fi & $\begin{array}{l}\text { Fuzzy-C-Means and } \\
\text { interpolation }\end{array}$ & $\begin{array}{c}\text { Rectangle area with } \\
\text { dimensions } 51.6 \times 20.4 \\
\mathrm{~m}^{2}\end{array}$ & $\begin{array}{l}\begin{array}{l}\text { Localization results } \\
\text { fingerprinting } \\
\text { yield } \\
0.23 \mathrm{~m}\end{array} \\
\text { improvement }\end{array}$ \\
\hline 6. & $\begin{array}{l}\text { V. Moghtadaiee, } \\
\text { et.al. }\end{array}$ & RSSI, Wi-Fi & $\begin{array}{l}\text { New interpolation method, } \\
\text { zone-based Weighted } \\
\text { Ring-based (WRB). }\end{array}$ & $\begin{array}{l}\text { 2D assumption: } 51 \times 18 \\
\mathrm{~m}^{2}\end{array}$ & $\begin{array}{l}\text { Decreases the error (up to } 57 \% \text { ) } \\
\text { between the measured and } \\
\text { reconstructed RSSI values. }\end{array}$ \\
\hline
\end{tabular}

The previous publications, mainly Wi-Fi-based RSSI as the Wi-Fi is more popular. However, if considering IoTbased indoor localization, we select the ZigBee standard devices in our case. The techniques and methods mentioned in Table 5 can vary from linear interpolation to path loss model-based RSSI data reconstruction. Most of the results of these publications and our proposed method can reduce the localization errors.

\section{5- Conclusions}

The database enhancement by applying interpolation and regression techniques to tackle one of the drawbacks of the fingerprint technique is presented. The actual measurement campaign in 2D and 3D environments was conducted, and RSSI values were used for the database fingerprint for both environments. We used a device for measurement based on IoT technology which cost-effective and straightforward implementation of ZigBee standards. The WSNs-based indoor localization is built and validated by some scenarios; the four target type placements, namely diagonal positions both left and right, the horizontal and vertical position for the $2 \mathrm{D}$ environment, while three scenarios tested 3D environment; clean, human body effects, and furniture effects. From all scenarios, almost all results agreed that enhancing the database to expand the database grids by artificial data can reduce localization prediction error. From all scenarios, the simple bilinear interpolation stood out as the best accuracy performance. For the $2 \mathrm{D}$ environment, it can reduce to $0.2 \mathrm{~m}$ error, and $0.13 \mathrm{~m}$ for the 3D environment, which is in this environment the grid size is $0.22 \mathrm{~m}(22 \mathrm{~cm})$. The performance improvement by replacing and adding the synthesis or artificial database can facilitate actual implementation. Thus, the sparsity issues in the fingerprint database can be tackled, and the offline database construction will be less burden.

Our research interest is in fingerprint database enhancement; for the next step, we consider applying machine or deep learning-based both supervised and unsupervised learning, both offline and online phase to increase the performance metric of indoor localization system and its flexibility and adaptability in the dynamic indoor environment.

\section{6- Declarations}

\section{6-1-Author Contributions}

Conceptualization, D.J.S.; methodology, D.J.S. and F.Y.M.A.; software, D.J.S. and F.Y.M.A.; validation, D.J.S., F.Y.M.A. and P.C.; formal analysis, D.J.S.; investigation, D.J.S.; resources, D.J.S., F.Y.M.A and P.C.; data curation, D.J.S.; writing - original draft preparation, D.J.S.; writing-review and editing, D.J.S., F.Y.M.A., P.C., and P.S.; visualization, D.J.S.; supervision, P.C. and P.S.; project administration, D.J.S. and P.C.; funding acquisition, P.C. and P.S. All authors have read and agreed to the published version of the manuscript. 


\section{6-2-Data Availability Statement}

The data presented in this study are available in article.

\section{6-3- Funding and Acknowledgements}

This work was supported by King Mongkut's Institute of Technology Ladkrabang (KMITL), Thailand.

\section{6-4- Conflicts of Interest}

The authors declare that there is no conflict of interests regarding the publication of this manuscript. In addition, the ethical issues, including plagiarism, informed consent, misconduct, data fabrication and/or falsification, double publication and/or submission, and redundancies have been completely observed by the authors.

\section{7- References}

[1] Marjani, Mohsen, Fariza Nasaruddin, Abdullah Gani, Ahmad Karim, Ibrahim Abaker Targio Hashem, Aisha Siddiqa, and Ibrar Yaqoob. "Big IoT Data Analytics: Architecture, Opportunities, and Open Research Challenges.” IEEE Access 5 (2017): 524761. doi:10.1109/ACCESS.2017.2689040.

[2] Bartoletti, Stefania, Andrea Conti, Davide Dardari, and Andrea Giorgetti. "5G Localization and Context-Awareness." In Whitepaper, 167-88, 2019.

[3] Xu, Guochang. GPS: Theory, Algorithms and Applications. GPS: Theory, Algorithms and Applications. Springer, (2007). doi:10.1007/978-3-540-72715-6.

[4] Yassin, Ali, Youssef Nasser, Mariette Awad, Ahmed Al-Dubai, Ran Liu, Chau Yuen, Ronald Raulefs, and Elias Aboutanios. "Recent Advances in Indoor Localization: A Survey on Theoretical Approaches and Applications." IEEE Communications Surveys and Tutorials 19, no. 2 (2017): 1327-46. doi:10.1109/COMST.2016.2632427.

[5] He, Suining, and S. H.Gary Chan. "Wi-Fi Fingerprint-Based Indoor Positioning: Recent Advances and Comparisons." IEEE Communications Surveys and Tutorials 18, no. 1 (2016): 466-90. doi:10.1109/COMST.2015.2464084.

[6] Yang, Chouchang, and Huai Rong Shao. "WiFi-Based Indoor Positioning.” IEEE Communications Magazine 53, no. 3 (2015): 150-57. doi:10.1109/MCOM.2015.7060497.

[7] Yan, Dongmei, Bing Kang, Hui Zhong, and Ruolin Wang. "Research on Positioning System Based on Zigbee Communication." In Proceedings of 2018 IEEE 3rd Advanced Information Technology, Electronic and Automation Control Conference, IAEAC 2018, 1027-30, 2018. doi:10.1109/IAEAC.2018.8577263.

[8] Jiang, Jehn Ruey, Hanas Subakti, and Hui Sung Liang. "Fingerprint Feature Extraction for Indoor Localization†.” Sensors 21, no. 16 (2021). doi:10.3390/s21165434.

[9] Yassin, Ali, Youssef Nasser, Mariette Awad, Ahmed Al-Dubai, Ran Liu, Chau Yuen, Ronald Raulefs, and Elias Aboutanios. "Recent Advances in Indoor Localization: A Survey on Theoretical Approaches and Applications." IEEE Communications Surveys and Tutorials 19, no. 2 (2017): 1327-46. doi:10.1109/COMST.2016.2632427.

[10] Song, Zhenlong, Gangyi Jiang, and Chao Huang. “A Survey on Indoor Positioning Technologies.” Communications in Computer and Information Science 164 CCIS (2011): 198-206. doi:10.1007/978-3-642-24999-0_28.

[11] Mehta, Rishika, Jyoti Sahni, and Kavita Khanna. "Internet of Things: Vision, Applications and Challenges." Procedia Computer Science 132 (2018): 1263-69. doi:10.1016/j.procs.2018.05.042.

[12] Carlos-Mancilla, Miriam, Ernesto López-Mellado, and Mario Siller. "Wireless Sensor Networks Formation: Approaches and Techniques.” Journal of Sensors (2016). doi:10.1155/2016/2081902.

[13] Willig, Andreas. "Recent and Emerging Topics in Wireless Industrial Communications: A Selection." IEEE Transactions on Industrial Informatics 4, no. 2 (May 2008): 102-124. doi:10.1109/tii.2008.923194.

[14] Yiu, Simon, Marzieh Dashti, Holger Claussen, and Fernando Perez-Cruz. "Wireless RSSI Fingerprinting Localization.” Signal Processing 131 (2017): 235-44. doi:10.1016/j.sigpro.2016.07.005.

[15] Mahler, Tom, and Ido Bayda. Product Design and Distance Measurement Using RSSI and LQI. Israel: Tel-Aviv University, 2013.

[16] Parameswaran, Ambili Thottam, Mohammad Iftekhar Husain, and Shambhu Upadhyaya. "Is RSSI a Reliable Parameter in Sensor Localization Algorithms - An Experimental Study.” In IEEE International Symposium on Reliable Distributed Systems, $1-5,2009$.

[17] Sadowski, Sebastian, and Petros Spachos. "RSSI-Based Indoor Localization with the Internet of Things.” IEEE Access 6 (2018): 30149-61. doi:10.1109/ACCESS.2018.2843325. 
[18] Li, Guoquan, Enxu Geng, Zhouyang Ye, Yongjun Xu, Jinzhao Lin, and Yu Pang. "Indoor Positioning Algorithm Based on the Improved Rssi Distance Model.” Sensors (Switzerland) 18, no. 9 (2018): 1-15. doi:10.3390/s18092820.

[19] Vo, Quoc Duy, and Pradipta De. "A Survey of Fingerprint-Based Outdoor Localization.” IEEE Communications Surveys and Tutorials 18, no. 1 (2016): 491-506. doi:10.1109/COMST.2015.2448632.

[20] Hatem, Elias, Sergio Fortes, Elizabeth Colin, Sara Abou-Chakra, Jean Marc Laheurte, and Bachar El-Hassan. "Accurate and Low-Complexity Auto-Fingerprinting for Enhanced Reliability of Indoor Localization Systems.” Sensors 21, no. 16 (2021): 5346. doi:10.3390/s21165346.

[21] Ji, Wenqing, Kun Zhao, Zhengqi Zheng, Chao Yu, and Shuai Huang. "Multivariable Fingerprints with Random Forest Variable Selection for Indoor Positioning System.” IEEE Sensors Journal, 2021, 1-1. doi:10.1109/jsen.2021.3103863.

[22] Nessa, Ahasanun, Bhagawat Adhikari, Fatima Hussain, and Xavier N. Fernando. "A Survey of Machine Learning for Indoor Positioning.” IEEE Access 8 (2020): 214945-65. doi:10.1109/ACCESS.2020.3039271.

[23] Wang, Liping, Saideep Tiku, and Sudeep Pasricha. "CHISEL: Compression-Aware High-Accuracy Embedded Indoor Localization with Deep Learning.” IEEE Embedded Systems Letters 0663, no. c (2021): 10-13. doi:10.1109/LES.2021.3094965.

[24] Chen, Guoliang, Xiaolin Meng, Yunjia Wang, Yanzhe Zhang, Peng Tian, and Huachao Yang. "Integrated WiFi/PDR/Smartphone Using an Unscented Kalman Filter Algorithm for 3D Indoor Localization.” Sensors (Switzerland) 15, no. 9 (2015): 24595-614. doi:10.3390/s150924595.

[25] Yan, Jun, Guowen Qi, Bin Kang, Xiaohuan Wu, and Huaping Liu. "Extreme Learning Machine for Accurate Indoor Localization Using RSSI Fingerprints in Multifloor Environments.” IEEE Internet of Things Journal 8, no. 19 (2021): $14623-37$. doi:10.1109/JIOT.2021.3071152.

[26] Gu, Zhuan, Zeqin Chen, Yuexing Zhang, Ying Zhu, Mingming Lu, and Ai Chen. "Reducing Fingerprint Collection for Indoor Localization.” Computer Communications 83 (2016): 56-63. doi:10.1016/j.comcom.2015.09.022.

[27] Bori, Marwa Mohammed, and Zahraa Ezzulddin Hussein. "Integration the Low Cost Camera Images with the Google Earth Dataset to Create a 3D Model.” Civil Engineering Journal 6, no. 3 (March 1, 2020): 446-458. doi:10.28991/cej-2020-03091482.

[28] Moghtadaiee, Vahideh, Seyed Ali Ghorashi, and Mohammad Ghavami. "New Reconstructed Database for Cost Reduction in Indoor Fingerprinting Localization.” IEEE Access 7 (2019): 104462-77. doi:10.1109/ACCESS.2019.2932024.

[29] Khalajmehrabadi, Ali, Nikolaos Gatsis, and David Akopian. "Structured Group Sparsity: A Novel Indoor WLAN Localization, Outlier Detection, and Radio Map Interpolation Scheme.” IEEE Transactions on Vehicular Technology 66, no. 7 (2017): 64986510. doi:10.1109/TVT.2016.2631980.

[30] Zayets, Alexandra, and Eckehard Steinbach. "Interpolation and Extrapolation of Multipath Fingerprints Using Virtual Transmitter Placement.” In IEEE International Conference on Communications, 2018. doi:10.1109/ICC.2018.8422206.

[31] Zuo, Jinbo, Shuo Liu, Hao Xia, and Yanyou Qiao. "Multi-Phase Fingerprint Map Based on Interpolation for Indoor Localization Using IBeacons.” IEEE Sensors Journal 18, no. 8 (2018): 3351-59. doi:10.1109/JSEN.2018.2789431.

[32] Li, Yanwei, Gaotao Shi, Xiaobo Zhou, Wenyu Qu, and Keqiu Li. "Reducing the Site Survey Using Fingerprint Refinement for Cost-Efficient Indoor Location.” Wireless Networks 25, no. 3 (2019): 1201-13. doi:10.1007/s11276-018-1711-6.

[33] Bi, Jingxue, Yunjia Wang, Hongji Cao, Hongxia Qi, Keqiang Liu, and Shenglei Xu. "A Method of Radio Map Construction Based on Crowdsourcing and Interpolation for Wi-Fi Positioning System.” In IPIN 2018 - 9th International Conference on Indoor Positioning and Indoor Navigation, (2018): 24-27. doi:10.1109/IPIN.2018.8533749.

[34] Zhu, Julie Yixuan, Anny Xijia Zheng, Jialing Xu, and Victor O.K. Li. "Spatio-Temporal (S-T) Similarity Model for Constructing WIFI-Based RSSI Fingerprinting Map for Indoor Localization.” In IPIN 2014 - 2014 International Conference on Indoor Positioning and Indoor Navigation, (2014): 678-84. doi:10.1109/IPIN.2014.7275543.

[35] Sun, Yongliang, Yu He, and Yang Yang. "Interpolation Method for Radio Map Establishment Based on RSS Clustering and Propagation Model Optimization.” In Proceedings - 2018 International Conference on Cyber-Enabled Distributed Computing and Knowledge Discovery, CyberC 2018, (2019): 451-54. doi:10.1109/CyberC.2018.00087.

[36] Assayag, Yuri, Horacio Oliveira, Eduardo Souto, Raimundo Barreto, and Richard Pazzi. "Indoor Positioning System Using Synthetic Training and Data Fusion.” IEEE Access 9 (2021): 115687-99. doi:10.1109/ACCESS.2021.3105188.

[37] Adiyatma, F Y M, A E Kurniawan, D J Suroso, and P Cherntanomwong. "Performance_comparison_of_several_publis.” Based Techniques for Indoor Localization Based on Received Signal Strength Indicator 7, no. 1 (2021): 40-53. doi:10.34818/ijoict.v7i1.550. 
[38] Wang, Xianmin, Zhikun Chen, Sihai Zhang, and Jinkang Zhu. "Super-Resolution Based Fingerprint Augment for Indoor WiFi Localization.” In 2020 IEEE Global Communications Conference, GLOBECOM 2020 - Proceedings, Vol. 2020-January, 2020. doi:10.1109/GLOBECOM42002.2020.9348146.

[39] Djosic, Sandra, Igor Stojanovic, Milica Jovanovic, Tatjana Nikolic, and Goran Lj Djordjevic. "Fingerprinting-Assisted UWBBased Localization Technique for Complex Indoor Environments.” Expert Systems with Applications 167 (2021): 114188. doi:10.1016/j.eswa.2020.114188.

[40] Zhang, Lingyan, and Hongyu Wang. "3D-WiFi: 3D Localization with Commodity WiFi.” IEEE Sensors Journal 19, no. 13 (2019): 5141-52. doi:10.1109/JSEN.2019.2900511.

[41] Wang, Xiaoyuan, Yongqing Guo, Chenglin Bai, Shanliang Liu, Shijie Liu, and Junyan Han. "The Effects of Weather on Passenger Flow of Urban Rail Transit." Civil Engineering Journal 6, no. 1 (January 1, 2020): 11-20. doi:10.28991/cej-202003091449.

[42] Tian, Xiaohua, Sujie Zhu, Sijie Xiong, Binyao Jiang, Yucheng Yang, and Xinbing Wang. "Performance Analysis of Wi-Fi Indoor Localization with Channel State Information." IEEE Transactions on Mobile Computing 18, no. 8 (2019): 1870-84. doi:10.1109/TMC.2018.2868680.

[43] Geok, Tan Kim, Khaing Zar Aung, Moe Sandar Aung, Min Thu Soe, Azlan Abdaziz, Chia Pao Liew, Ferdous Hossain, Chih P. Tso, and Wong Hin Yong. "Review of Indoor Positioning: Radio Wave Technology.” Applied Sciences (Switzerland) 11, no. 1 (2021): 1-44. doi:10.3390/app11010279.

[44] Cheng, Yen Kai, Hsin Jui Chou, and Ronald Y. Chang. "Machine-Learning Indoor Localization with Access Point Selection and Signal Strength Reconstruction.” IEEE Vehicular Technology Conference 2016-July (2016): 2016-. doi:10.1109/VTCSpring.2016.7504333.

[45] Cherntanomwong, Panarat, and Dwi Joko Suroso. "Indoor Localization System Using Wireless Sensor Networks for Stationary and Moving Target.” Conf. Information, Commun. Signal Process 1 (2012): 1-5. doi:10.1109/icics.2011.6173554.

[46] Teukolsky, Saul A., Brian P. Flannery, W. H. Press, and W. T. Vetterling. "Numerical recipes in C." SMR 693, no. 1 (1992): 59-70.

[47] Kiusalaas, Jaan. "Numerical Methods in Engineering with Python 3." In Numerical Methods in Engineering with Python 3, 3 , 2013. doi:10.1017/cbo9781139523899.

[48] Ostertagová, Eva. "Modelling Using Polynomial Regression.” Procedia Engineering 48 (2012): 500-506. doi:10.1016/j.proeng.2012.09.545.

[49] Suroso, Dwi Joko, Panarat Cherntanomwong, Pitikhate Sooraksa, and Jun Ichi Takada. "Location Fingerprint Technique Using Fuzzy C-Means Clustering Algorithm for Indoor Localization.” In IEEE Region 10 Annual International Conference, Proceedings/TENCON, 88-92, 2011. doi:10.1109/TENCON.2011.6129069.

[50] Suroso, Dwi Joko, Panarat Cherntanomwong, Pitikhate Sooraksa, and Jun Ichi Takada. "Fingerprint-Based Technique for Indoor Localization in Wireless Sensor Networks Using Fuzzy C-Means Clustering Algorithm.” In 2011 International Symposium on Intelligent Signal Processing and Communications Systems: "The Decade of Intelligent and Green Signal Processing and Communications”, ISPACS 2011, 1-5, 2011. doi:10.1109/ISPACS.2011.6146167.

[51] Cherntanomwong, Panarat, and Dwi Joko Suroso. "Indoor Localization System Using Wireless Sensor Networks for Stationary and Moving Target.” Conf. Information, Commun. Signal Process 1 (2012): 1-5. doi:10.1109/icics.2011.6173554.

[52] Suroso, Dwi Joko, Panarat Cherntanomwong, Pitikhate Sooraksa, and Jun Ichi Takada. "Location Fingerprint Technique Using Fuzzy C-Means Clustering Algorithm for Indoor Localization.” In IEEE Region 10 Annual International Conference, Proceedings/TENCON, 88-92, 2011. doi:10.1109/TENCON.2011.6129069.

[53] Cherntanomwong, Panarat, Jun Ichi Takada, and Hiroyuki Tsuji. "Signal Subspace Interpolation from Discrete Measurement Samples In Constructing a Database for Location Fingerprint Technique.” IEICE Transactions on Communications E92-B, no. 9 (2009): 2922-30. doi:10.1587/transcom.E92.B.2922.

[54] Suroso, Dwi Joko, Alvin S.H. Rudianto, Muhammad Arifin, and Singgih Hawibowo. "Random Forest and Interpolation Techniques for Fingerprint-Based Indoor Positioning System in Un-Ideal Environment." International Journal of Computing and Digital Systems 10, no. 1 (2021): 701-713. doi:10.12785/IJCDS/100166.

[55] Manirabona, Audace, and Lamia Chaari Fourati. "A Kriged Fingerprinting for Wireless Body Area Network Indoor Localization." Wireless Personal Communications 80, no. 4 (2015): 1501-15. doi:10.1007/s11277-014-2095-2.

[56] King, Thomas, Thomas Haenselmann, and Wolfgang Effelsberg. "On-Demand Fingerprint Selection for 802.11-Based Positioning Systems.” In 2008 IEEE International Symposium on a World of Wireless, Mobile and Multimedia Networks, WoWMoM2008, 2008, 2008. doi:10.1109/WOWMOM.2008.4594839. 